\title{
Desgaste dental y hábitos dietarios en poblaciones arqueológicas del centro de Argentina
}

4 Claudina V. González" y Mariana Fabra*

\section{Resumen}

El objetivo del presente trabajo es aportar al conocimiento sobre las estrategias de subsistencia y dieta de las poblaciones que habitaron el centro de Argentina, particularmente el actual territorio de la provincia de Córdoba en el Holoceno tardío, a partir del análisis macroscópico del patrón y el grado de desgaste dental. La muestra analizada está compuesta por 61 individuos adultos (19 femeninos y 42 masculinos) que provienen de 36 sitios arqueológicos de dos regiones geográficas, denominadas Sierras y Llanuras. Se cuenta con información radiocarbónica para 52 individuos, que permite ubicarlos en un rango entre los $4058 \pm 89$ y los $370 \pm 15{ }^{14} \mathrm{C}$ años AP. Se registró de forma macroscópica el grado, patrón y forma del desgaste dental sobre 1.021 piezas dentales y luego se calcularon prevalencias según el sexo, la edad de muerte, la región (Sierras y Llanuras) y el período cronológico (previo a 1200 años AP, posterior a 1200 años AP). Se aplicaron análisis estadísticos (Chi-cuadrado) para evaluar la significación estadística de las diferencias observadas. Los resultados indican la existencia de diferencias regionales y temporales, tanto en la forma como en el grado del desgaste dental de estas poblaciones. Consideramos que estas diferencias estarían relacionadas a diferentes formas y técnicas de preparación de los alimentos antes que a cambios dietarios.

\section{Tooth wear and dietary habits in archaeological populations of the center of Argentina}

\begin{abstract}
The aim of this paper is to contribute to the knowledge of subsistence strategies and dietary habits of the human populations that inhabited the central region of Argentina during the Late Holocene through the evaluation of physiological stress indicators (pattern and degree of tooth wear). The samples correspond to a total of 61 individuals (19 females and 42 males) recovered from 36 archaeological sites located

* Instituto de Antropología de Córdoba (IDACOR), Facultad de Filosofía y Humanidades, Universidad Nacional de Córdoba (UNC) - CONICET. Av. Hipólito Yrigoyen 174 (CP X50ooJHO), Córdoba Argentina. E-mail: cvictoriagonzalez22@hotmail.com; marianafabra@gmail.com
\end{abstract}

Recibido:

24 de abril de 2017

Aceptado:

30 de agosto de 2017

Palabras Clave

Desgaste dental Dieta

Holoceno tardío

Córdoba

\section{Keywords}

Tooth wear

Diet

Late Holocene

Córdoba 
in two geographic and environmental subregions of the province of Córdoba -Central Highlands and Eastern Lowlands- and dated by AMS between $4058 \pm 89$ and $370 \pm 15$ ${ }^{14} \mathrm{C}$ years BP. A macroscopic record of the degree, pattern and shape of tooth wear was performed on 1,021 dental pieces, and their prevalence was calculated according to sex, age of death, region (Central Highlands and Eastern Lowlands), and chronological period (before 1200 years BP and after 1200 years BP). Statistical analyzes (Chi-square tests) were applied to test the existence of significant differences between groups. The results indicate the existence of regional and temporal differences in the form and degree of tooth wear. We consider that these differences could be related to different forms and techniques of food preparation rather than to dietary differences.

\section{Introducción}

Los dientes son uno de los elementos mejor conservados a través del tiempo. Sus características físicas y químicas le confieren una muy baja susceptibilidad a la degradación postdepositacional en contextos arqueológicos, permitiendo así una mayor conservación respecto a las otras partes esqueletales (Hillson, 1996). Desde el momento en que se produce la erupción dental, comienza un proceso de envejecimiento natural y continuo, derivado del uso normal del aparato masticatorio (Alt y Pichtler, 1998). La pérdida de tejido dentario durante la vida de un individuo se denomina desgaste dentario (Cruwys, 1989). Comienza a actuar en principio sobre el esmalte y luego sobre la dentina, llegando a afectar la estructura vital de la pieza dental, ya en casos extremos la exposición de la pulpa y los tejidos blandos del diente son afectados, produciéndose necrosis pulpar y la posterior pérdida del diente (Hillson, 1996).

Desde el punto de vista clínico, es fundamental discriminar cuáles son las causas que provocan la pérdida del tejido dental y los diferentes tipos de desgaste que están actuando (Alt y Pilcher, 1998), aunque desde el punto de vista bioarqueológico es difícil discriminar cuál de ellas es la causa del desgaste registrado. Básicamente existen tres tipos de desgaste del tejido dental (Cruwys, 1989). La atrición, provocada por el contacto entre dientes vecinos o antagonistas (Cruwys, 1989) que suele estar relacionada íntimamente con las fuerzas masticatorias y la dureza de los alimentos o incluso el bruxismo (Campillo, 2001). La abrasión, producida por la acción de partículas exógenas (Cruwys, Robb y Smith, 1992; Hillson, 1996) proveniente de granos o sustancias ajenas a la alimentación (Bonfiglioli, Mariotti, Facchini, Belcastro y Condemi, 2004; Larsen, Teaford y Sandford, 1998; Lukacs y Pastor, 1998; Molnar, 2008; Neto Wasterlain, 2006). Por último, la erosión, provocada por la disolución química del esmalte inducida por los ácidos de ciertas bebidas carbonatadas, alcohólicas, jugos de frutas, ácidos estomacales en procesos de regurgitación o vómitos recurrentes (Soames y Southam, 2005).

Son varios los factores que pueden influir en el desgaste, desde la estructura dentaria y del esmalte, la higiene oral, las prácticas terapéuticas, los traumas dentarios, la maloclusión, la erupción dentaria y también factores externos como la dieta, las prácticas culturales y la edad (Clarke y Hirsch, 1991; Flensborg, 2012; Hinton, 1981; Smith, 1984; Turner II y Machado, 1983). Los tipos de alimentos consumidos, la forma de preparación, la cocción, entre otros, también son factores a considerar. En relación con esto, varios estudios han desarrollado comparaciones entre poblaciones cazadoras recolectoras y agricultoras llegando a encontrar diferencias significativas no sólo en el grado sino en el patrón del desgaste (Hinton, 1981; Littleton y Frohlich, 1993; Molnar, 1971; Smith, 1984). En general, las poblaciones cazadoras-recolectoras presentan mayor desgaste oclusal debido a la mayor dureza de los alimentos (Deter, 2009; Molnar, 1971; Smith, 1984); en cambio, las sociedades de economía agrícola tienden a presentar 
menor degaste dental (Smith, 1984). Por otro lado, se ha observado que en sociedades con un mismo tipo de dieta pero que procesan y cocinan sus alimentos de forma distinta, los grados de desgaste dental también difieren (Clement y Hillson, 2013; Delgado-Darías, Velasco-Vázquez, Arnay de Larosa, Martín-Rodríguez y GonzálezReimers, 2005; Powell, 1985). Otros factores que inciden en el desgaste dental son los hábitos culturales, aunque estas influencias sobre los dientes son variadas y difíciles de determinar (Cruwys, 1989). Las modificaciones culturales pueden ser individuales o colectivas, e incluyen la utilización de los dientes como "tercera mano" y prácticas de modificación relacionadas a ideología o estética personal (Alt y Pichtler, 1998). Sin embargo, las mayores exigencias mecánicas sobre los dientes son las que involucran la utilización de la dentición en hábitos no masticatorios (Bonfiglioli et al., 2004; Lozano, 2005; Lukacs y Pastor, 1988; Molnar, 1972).

Diversos estudios han demostrado que tanto poblaciones con estrategias de subsistencia basadas en la caza y la recolección como comunidades agricultoras pueden presentar pronunciados grados de desgaste dental (Hinton, 1981; Smith, 1984). Sin embargo, las causas que lo originan son diferentes, en algunos casos relacionadas con cuestiones ambientales y en otros con tipos de subsistencia (Bernal, Novellino, González y Pérez, 2007; L'Heureux, 2002). Estudios comparativos tendientes a analizar el patrón de desgaste dental entre poblaciones con distintos modos de subsistencia han encontrado que en poblaciones con economías basadas en la caza y recolección la atrición es más pronunciada, con la presencia de formas planas principalmente en la dentición anterior (Hinton, 1981; Smith, 1984). Por el contrario, en poblaciones agrícolas el desgaste tiende a disminuir en la dentición anterior y a incrementarse en los dientes posteriores, presentando formas cóncavas (Hinton, 1981; Molnar, 1971; Smith, 1984). Estas diferencias en las formas se deben principalmente a que los grupos agricultores emplean técnicas de cocción y preparación que tienden a disminuir la dureza y la fibrosidad de los alimentos (Gheggi, 2012; L'Heureux, 2000), mientras que en el caso de las sociedades cazadoras-recolectoras la textura de los alimentos y las formas en las que son preparados generarían un mayor estrés masticatorio (Flensborg, 2012; Gómez Otero y Novellino, 2011; Luna y Aranda, 2014; Smith, 1984). Otros autores relacionan el mayor desgaste en la dentición anterior de los cazadores-recolectores a la mayor utilización de la dentición en actividades paramasticatorias, como lo es la fabricación de herramientas y utensilios (Hinton, 1981; Molnar, 2008). En relación con esto, investigaciones realizadas sobre muestras dentales de poblaciones cazadoras recolectoras de la región Pampeana (Luna y Aranda, 2014) y el este Pampeano-Patagónico sugieren que la mayor presión masticatoria registrada en los individuos femeninos de esa población (Flensborg, 2011) podría estar relacionada con la utilización de la dentición en actividades cotidianas como la preparación de alimentos o la elaboración de utensilios, entre otros. Sin embargo, en estas poblaciones cazadoras-recolectoras se registra una disminución de los grados de desgaste dental hacia momentos tardíos (Bernal et al., 2007; Gomez Otero y Novellino, 2011). En cuanto al patrón de desgaste, las referencias a su registro a nivel poblacional aún son escasas en nuestro país: L’Heureux (2002) ha relevado este indicador en relación con el tipo de dieta de poblaciones con economía cazadorarecolectora de la región Pampeana, observando un predominio de patrones cóncavos en molares y redondeados en la dentición anterior. En la región central de Argentina, particularmente el área ocupada por las Sierras Centrales y llanuras pampeanas hacia el este en la provincia de Córdoba, no se han realizado hasta el momento estudios de desgaste dental a nivel poblacional.

Las investigaciones arqueológicas realizadas en esta región sugieren una ocupación humana que se inicia hace 10.000 años y el posterior desarrollo de diversas estrategias y adaptaciones al ambiente por parte de las poblaciones (Laguens, 1999; Laguens y Bonnin, 2009). A lo largo del Holoceno, estas poblaciones desarrollaron tecnologías y modalidades de explotación de los recursos de manera eficaz, siendo la caza y la 
1. Sitio C.Pun. 39. (Valle de Punilla) caracterizado como un sitio multipropósito de ocupación estacional, se obtuvieron macrorestos de Phaseulus vulgaris, fechados en $525 \pm 36$ años AP (Medina, 2009). Quebrada del Real 1- Pampa de Achala- sitio en pampas de altura, fechado en $2950 \pm 90$ años AP (López et al., 2015), se recuperaron restos de Chenopodium spp. y/o Amaranthus

spp.

2. Se citan algunos fragmentos de la crónica de Sotelo de Narváez de 1583, y la Relación Anónima de 1573, donde se describe la producción de algunos cultígenos: “...es tierra fértil de mucho maíz y frisoles $y$ maní y camote y sapallos..." (Sotelo Narváez, 1583), “...sembraban quinoa y sapallos y mays y en el dicho sitio están cantidades de morteros de piedra que los indios llamaban Tacanas en que los asistían allí molían sus comidas..." (Huamiltocto, en Piana de Cuesta, recolección las estrategias de subsistencia principales durante varios milenios. A inicios de la era cristiana, nuevas tecnologías tales como la producción cerámica, un estilo de vida sedentario y la incorporación de las prácticas agrícolas de manera complementaria a las estrategias de caza y recolección, configuraron un nuevo modo de vida que identificó a estas poblaciones (Laguens y Bonnin, 2009). Este modo de vida no fue homogéneo en toda la región de la provincia de Córdoba: las distintas adaptaciones a los ambientes serranos y de llanura, sumadas a características de las técnicas de producción cerámica y otros bienes materiales que marcaron particularidades regionales, hace pensar en la existencia de una diversidad que contradice a la unidad cultural postulada por los cronistas españoles (Laguens, 1999; Laguens y Bonnin, 2009).

Los distintos sitios arqueológicos y sus características en cuanto a registro material nos permiten tener una imagen de la particularidad de los modos de vida de las poblaciones que se asentaron allí en distintos momentos del Holoceno. Así, observamos que las manifestaciones culturales desarrolladas en Sierras difieren en algunos aspectos en la región Llanuras, siendo lo más notable la presencia -hacia momentos finales del Holoceno tardío- de sitios residenciales caracterizados por la existencia de casas-pozo, cerámica con incisiones o surcos rítmicos, estatuillas cerámicas, arte rupestre, gran cantidad de materiales líticos y la presencia de grandes sitios de molienda, posiblemente comunitarios. En cuanto a las prácticas mortuorias, parecen predominar los entierros primarios dobles con los miembros hiperflexionados hacia la derecha o la izquierda y el cuerpo orientado en sentido Norte-Sur (Fabra, Salega y González, 2009). En cambio en Llanuras se hace evidente la presencia de cerámica con improntas de cestería muy similar a las halladas en la región del Litoral y la escasa presencia de materiales líticos, al tiempo que se registran sitios residenciales con gran cantidad de depósitos subterráneos denominados "Hornillos", característica que también se encuentra en sitios ubicados al Norte de la provincia de Córdoba. Allí, los entierros humanos se caracterizan por ser inhumaciones primarias simples, en posición extendida, con orientación Sur-Norte.

Respecto a estudios arqueológicos vinculados al uso de recursos vegetales, la evidencia más directa de prácticas de cultivo, o mejor dicho del consumo y manejo de recursos producidos, ha sido aportada por la arqueobotánica. Pastor (2007) y Medina, López y Berberián (2009) han recuperado evidencia de varios cultígenos en sitios ubicados en la región serrana, como maíz (Zea mays), calabaza (Cucurbita sp.), porotos (Phaseolus vulgaris) y, para momentos previos, también quínoa y amaranto (Chenopodium spp./ Amaranthus spp) tanto en forma de macrorrestos carbonizados como de microrrestos vegetales asociados a sedimentos, instrumentos de molienda y tiestos cerámicos (López, Medina y Rivero, 2015; Medina et al., 2009; Pastor y López, 2010). La presencia de maíz, porotos y calabazas ha sido fechada hacia momentos finales del Holoceno ${ }^{1}$ (López et al., 2015), mientras que la presencia y el posible manejo de especies como Chenopodium spp. y/o Amaranthus spp., recuperadas en materiales de molienda ha sido datada hacia momentos iníciales del Holoceno tardío en sitios no aptos para el cultivo. Por otra parte, las fuentes etnohistóricas han referido la presencia de maizales y chacras cerca de las casas o dispersas en distintos lugares de las Sierras, lejos de los lugares de asentamiento. Son varias las fuentes del siglo XVI y XVII que hacen referencia al cultivo de productos como maíz, zapallo, poroto e incluso quínoa ${ }^{2}$. Por otro lado, en la región Llanuras, específicamente en cercanías a la laguna Mar Chiquita, las investigaciones arqueológicas relacionadas a la incorporación de la agricultura son escasas y no nos informan sobre esta práctica, por lo que debemos recurrir a la información brindada por los isótopos estables, que indican una mayor dependencia de recursos recolectados, tanto de origen animal como vegetal (Fabra y González, 2016; González, 2016).

Para fines del Holoceno tardío, el registro arqueológico regional hace suponer que entre 1000 y 500 años AP se habría producido un desmejoramiento en la calidad de vida de las poblaciones, así como un crecimiento demográfico y una explotación más intensiva 
de los espacios, lo que habría generado una presión sobre los recursos disponibles en los distintos ambientes (Laguens y Bonnin, 2009). Estudios bioarqueológicos acerca de la salud y los niveles de actividad física dan cuenta de un incremento en la frecuencia de patologías infecciosas orales y marcadores entésicos hacia fines del Holoceno tardío (Fabra y González, 2015; Fabra, González, Salega, 2012a; Fabra, Nores, Salega y González, 2014a; González y Fabra, 2011; Salega y Fabra, 2013). Este proceso se habría acentuado hacia el 500 AP, derivando en situaciones de circunscripción ambiental y social que habrían conducido al surgimiento de potenciales conflictos sociales por nuevos territorios para el asentamiento y la explotación de recursos (Laguens y Bonnin, 2009).

En este marco, el objetivo del presente trabajo es contribuir al estudio de los modos de vida de los grupos humanos que habitaron las sierras y llanuras de la provincia de Córdoba en el Holoceno tardío, a partir del análisis del desgaste dental. Se analizan indicadores dentales -grado, patrón y forma del desgaste dental- con el objetivo de abordar tanto cuestiones vinculadas con la dieta de estas poblaciones, la importancia de los alimentos cultivados como el maíz (Zea mays) o los recolectados (frutos de algarroba, mistol o chañar), considerando la dureza y fibrosidad de los alimentos, así como con prácticas alimenticias, tipos de moliendas y preparado de los alimentos, y finalmente la posible utilización de la dentición en actividades paramasticatorias.

\section{Área de estudio}

Los individuos analizados en el presente trabajo proceden de sitios arqueológicos ubicados entre los $30^{\circ}$ y $35^{\circ}$ de latitud Sur y los $62^{\circ}$ y $66^{\circ}$ de longitud Oeste, abarcando los cordones montañosos de las provincias de Córdoba y San Luis, así como las llanuras orientales y occidentales aledañas. Esta región presenta contrastes entre sierras y llanuras: la planicie oriental, correspondiente a la llanura pampeana, y las sierras en el Oeste. Entre las dos principales unidades orográficas -las Sierras de Córdoba y las de San Luis- se desarrolla una segunda llanura, o planicie occidental, separando ambos cordones mediante un amplio valle intermontano. Desde un punto de vista fitogeográfico, la provincia de Córdoba representa dentro del dominio Chaqueño, a las provincias Chaqueña, del Espinal y Pampeana, que la atraviesan como bandas longitudinales en sentido Noreste-Sudoeste (Cabrera, 1976). En el sector norte de la región existen dos grandes humedales: la Laguna de Mar Chiquita o Mar de Ansenuza, en el Noreste, y las Salinas Grandes, en el Noroeste (Figura 1).

\section{Materiales y métodos}

Se estudió la dentición permanente de 61 individuos adultos, 42 de sexo masculino y 19 de sexo femenino, provenientes de 36 sitios arqueológicos localizados en las regiones de Sierras (32 individuos) y Llanuras (28 individuos) (Figura 1). Se relevó un total de 1.021 piezas dentales. Se cuenta con información radiocarbónica mediante fechados AMS para 52 individuos, los cuales se ubican en un rango temporal que abarca desde los $4058 \pm 89$ hasta los $370 \pm 15{ }^{14} \mathrm{C}$ años AP. El resto de los individuos puede ser adscripto al Holoceno tardío, en función de la información arqueológica disponible (Tabla 1).

Se tuvieron en cuenta dos criterios para proceder al agrupamiento de las muestras, uno geográfico, diferenciando dos grandes subregiones para la provincia de Córdoba -Sierras y Llanuras- cada una de ellas caracterizadas por similitudes y diferencias ambientales, geográficas y arqueológicas, y otro cronológico, que nos permitirá analizar la diversidad temporal de la dieta y poner a prueba la hipótesis de continuidad o cambio en el tipo de alimentos consumidos - previo a 1200 años AP (ca. 4000-1200 años AP) y posterior a 1200 años AP (1200-300 años AP). 


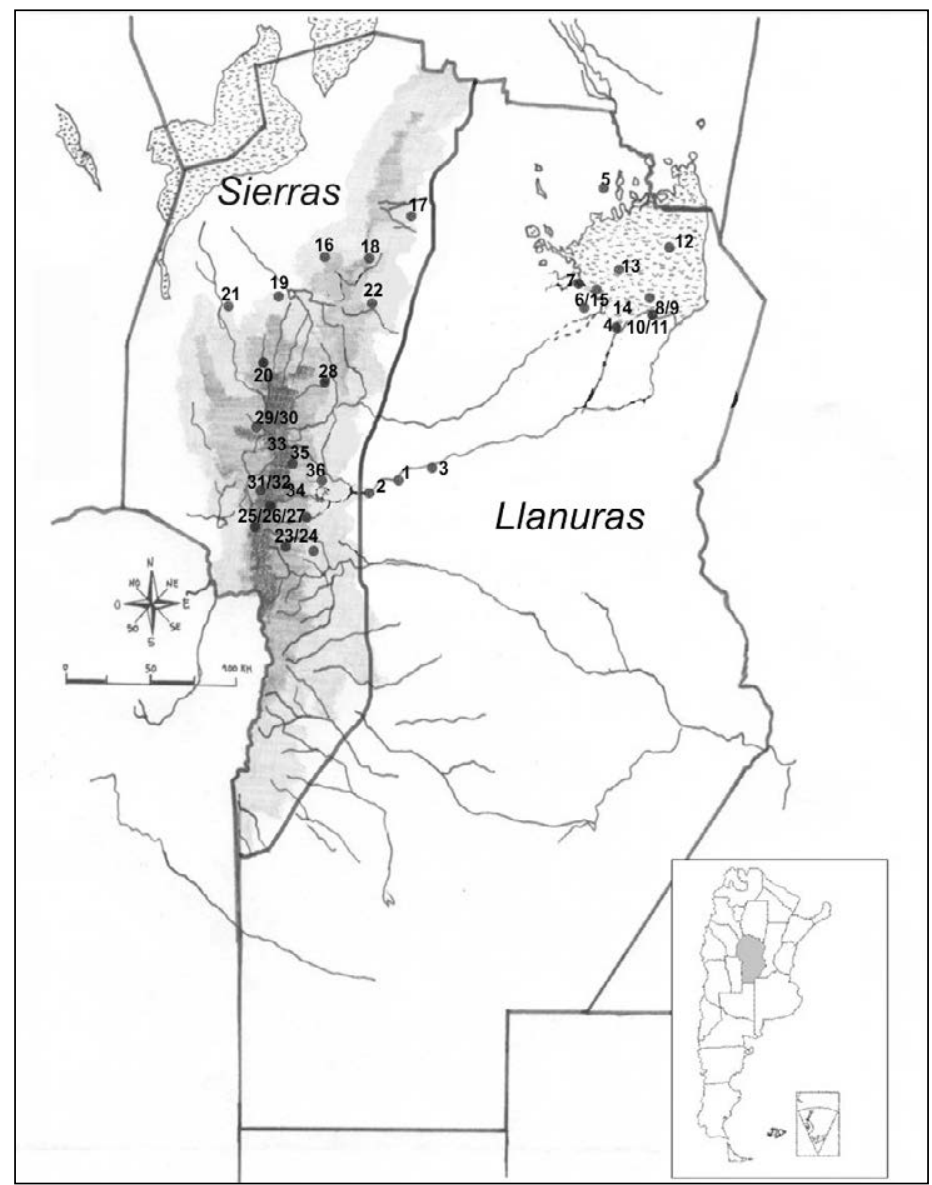

Figura 1. Sitios arqueológicos considerados en este estudio, agrupados por subregiones geográficas de la provincia de Córdoba. Llanuras: 1) Costasacate, 2) Cosme, 3) Rincón. 4) El Diquecito, 5) Laguna de la Sal, 6) La Para, 7) Ea. La Elisa 8) Orihuela, 9) Isla Orihuela, 10) Colonia Muller, 11) Miramar, 12) Isla Tigre, 13) El Mistolar, 14) Laguna del Plata, 15) Mar Chiquita. Sierras: 16) Rosca Yaco, 17) Cerro Colorado, 18) Nunsacat, 19) Ischilin, 20) San Esteban, 21) Charquina, 22) El Vado, 23) Los Molinos, 24) Potrero de Garay, 25) Quillinzo, 26) Va. Rumipal, 27) Banda Meridional del Lago, 28) La Granja, 29) Cuesta Blanca, 30) Ecoterra. 31) Loma Bola, 32) Guasmara, 33) Copina, 34) Santa Rosa de Calamuchita, 35) Constantinopla, 36) Potrerillo de Larreta.

Dicha periodificación se justifica en que ambos momentos estarían representando cambios en las estrategias de subsistencia y, de forma más general, en el modo de vida de estas comunidades (Fabra et al., 2014a). Las diferencias observadas en las prevalencias de indicadores de salud bucal y dieta (Fabra y González, 2015; González, 2011, 2016) así como también los marcadores de actividad física (Salega y Fabra, 2013) sugieren cambios no sólo en el tipo de estrategias de subsistencia desarrolladas en cada una de las regiones y las actividades realizadas por las personas, sino también en las cronologías de ocurrencia de estos procesos. El análisis en conjunto de las prevalencias de indicadores dentales y los valores de isótopos de carbono $\left({ }^{13} \mathrm{C}\right)$ hacia fines del Holoceno tardío nos estarían sugiriendo que las poblaciones de las Sierras habrían incorporado recursos agrícolas con mayor intensidad que los de las Llanuras (Fabra y González, 2016). En esta última región se registra una disminución en la prevalencia de caries hacia el Holoceno tardío final, lo cual podría estar relacionado con el aumento de los grados de desgaste dental y con las perdidas dentales antemortem registradas en esta región, indicadores que pueden eliminar o borrar la presencia de caries. Por otro lado los valores isotópicos del carbono se mantienen relativamente constantes a lo largo del tiempo, lo que podría sugerirnos que en Llanuras se estaban sucediendo cambios en las practicas de cocción o almacenamiento 


\begin{tabular}{|c|c|c|c|c|c|c|c|}
\hline Región & Sitio & $\begin{array}{l}\text { Fechados }{ }^{14} \mathrm{C} \\
\text { (años AP) }\end{array}$ & $\begin{array}{c}\text { Fechados calibrados } 2 \\
\text { sigmas }\end{array}$ & $\begin{array}{l}\text { Código de } \\
\text { laboratorio }\end{array}$ & Sexo & Edad & $\begin{array}{l}\text { Material } \\
\text { fechado }\end{array}$ \\
\hline LL & Orihuela & $1045 \pm 15$ & $870 \mathrm{AD}(95,4 \%) 935 \mathrm{AD}$ & $\mathrm{UCl}_{39101}$ & $\mathrm{~F}$ & A & Diente \\
\hline LL & Mar Chiquita & $1191 \pm 58$ & $640 \mathrm{AD}(95,4 \%) 880 \mathrm{AD}$ & MTC-14024 & $M$ & AMD & Diente \\
\hline LL & El Diquecito & $1192 \pm 40$ & $670 \mathrm{AD}(95,4 \%) 840 \mathrm{AD}$ & MTC-13247 & M & AM & Hueso \\
\hline LL & Lag. del Plata & $1241 \pm 57$ & $590 \mathrm{AD}(95,4 \%) 830 \mathrm{AD}$ & $\mathrm{AA} 102654$ & $\mathrm{~F}$ & AMD & Diente \\
\hline LL & Colonia Muller & $1585 \pm 15$ & $330 \mathrm{AD}(95,4 \%) 395 \mathrm{AD}$ & $\mathrm{UCl}_{39102}$ & $\mathrm{~F}$ & AMD & Diente \\
\hline LL & Est. La Elisa & $1890 \pm 49$ & $40 \mathrm{AC}(95,4 \%) 160 \mathrm{AD}$ & $\mathrm{AA}_{102656}$ & M & AJ & Hueso \\
\hline LL & El Diquecito & $1911 \pm 59$ & $90 \mathrm{AC}(95,4 \%) 160 \mathrm{AD}$ & MTC-14023 & M & $A$ & Hueso \\
\hline LL & El Mistolar & $2282 \pm 47$ & $430 \mathrm{AC}(95,4 \%) 230 \mathrm{AC}$ & $\mathrm{AA}_{104743}$ & M & AJ & Diente \\
\hline LL & El Diquecito & $2331 \pm 46$ & $480 \mathrm{AC}(95,4 \%) 28 \mathrm{o} A C$ & AA93745 & $M$ & A & Hueso \\
\hline LL & El Diquecito & $2400 \pm 47$ & $550 \mathrm{AC}(95,4 \%) 350 \mathrm{AC}$ & AA93740 & M & AM & Hueso \\
\hline LL & El Diquecito & $2562 \pm 47$ & $710 \mathrm{AC}(95,4 \%) 510 \mathrm{AC}$ & AA93742 & M & AMD & Hueso \\
\hline LL & La Para & $370 \pm 15$ & $1546 \mathrm{AD}(95,4 \%) 1610 \mathrm{AD}$ & UCl 39099 & M & A & Hueso \\
\hline LL & Est. La Elisa & $4058 \pm 89$ & $2290 \mathrm{AC}(95,4 \%) 1930 \mathrm{AC}$ & $\mathrm{AA}_{102655}$ & M & A & Diente \\
\hline LL & Orihuela & $487 \pm 45$ & $1370 \mathrm{AD}(95,4 \%) 1560 \mathrm{AD}$ & $\mathrm{AA}_{102657}$ & $\mathrm{~F}$ & AMD & Hueso \\
\hline $\mathrm{LL}$ & Rincón & $520 \pm 15$ & $1395 \mathrm{AD}(95,4 \%) 1460 \mathrm{AD}$ & $\mathrm{UCl} 22285$ & M & A & - \\
\hline LL & El Diquecito & $537 \pm 57$ & $1290 \mathrm{AD}(95,4 \%) 1530 \mathrm{AD}$ & MTC-14022 & M & AMD & Hueso \\
\hline LL & Costasacate & $616 \pm 58$ & $1210 \mathrm{AD}(95,4 \%) 1450 \mathrm{AD}$ & MTC-14026 & $\mathrm{F}$ & AM & Hueso \\
\hline LL & Lag. de la Sal & $623 \pm 42$ & $1240 \mathrm{AD}(95,4 \%) 1420 \mathrm{AD}$ & MTC-13255 & $M$ & AMD & Diente \\
\hline LL & Orihuela & $664 \pm 150$ & $950 \mathrm{AD}(95,4 \%) 1600 \mathrm{AD}$ & $\mathrm{AA} 104741$ & M & A & Hueso \\
\hline LL & El Mistolar & $690 \pm 85$ & $1080 \mathrm{AD}(95,4 \%) 1430 \mathrm{AD}$ & MTC-12805 & $\mathrm{F}$ & A & Diente \\
\hline LL & El Diquecito & $698 \pm 42$ & $1160 \mathrm{AD}(95,4 \%) 1340 \mathrm{AD}$ & AA93743 & M & A & Hueso \\
\hline LL & El Diquecito & $750 \pm 85$ & $1020 \mathrm{AD}(95,4 \%) 1370 \mathrm{AD}$ & MTC-12807 & M & A & Diente \\
\hline LL & Orihuela & $761 \pm 41$ & $1100 \mathrm{AD}(95,4 \%) 128 \mathrm{o} A \mathrm{D}$ & AA93746 & M & AJ & Diente \\
\hline $\mathrm{LL}$ & El Diquecito & $937 \pm 150$ & $700 \mathrm{AD}(95,4 \%) 1350 \mathrm{AD}$ & MTC-13214 & $\mathrm{F}$ & AMD & Hueso \\
\hline LL & Isla Tigre & $958 \pm 35$ & $920 \mathrm{AD}(95,4 \%) 1070 \mathrm{AD}$ & MTC-14847 & M & AMD & Hueso \\
\hline LL & Cosme & $\mathrm{S} / \mathrm{F}$ & - & - & $M$ & AJ & - \\
\hline LL & Rincón & $S / F$ & - & - & $\mathrm{F}$ & A & - \\
\hline LL & Rincón & $S / F$ & - & - & M & $A$ & - \\
\hline $\mathrm{S}$ & Ptllo de Larreta & $1067 \pm 77$ & $720 \mathrm{AD}(95,4 \%) 1040 \mathrm{AD}$ & $\mathrm{AA} 102660$ & $\mathrm{~F}$ & A & Diente \\
\hline S & Cuesta Blanca & $1080 \pm 40$ & $780 \mathrm{AD}(95,4 \%) 950 \mathrm{AD}$ & MTC-13249 & M & A & Diente \\
\hline $\mathrm{S}$ & La Granja & $1280 \pm 20$ & $625 \mathrm{AD}(95,4 \%) 710 \mathrm{AD}$ & $\mathrm{UCl} 22282$ & M & A & Diente \\
\hline S & Ecoterra & $1881 \pm 38$ & $10 \mathrm{AC}(95,4 \%) 120 \mathrm{AD}$ & $\mathrm{AA} 104742$ & M & A & Diente \\
\hline$S$ & El Vado & $2156 \pm 86$ & $390 \mathrm{AC}(95,4 \%) 30 \mathrm{AC}$ & MTC-128o8 & M & AMD & Diente \\
\hline S & Copina & $2707 \pm 61$ & $880 \mathrm{AC}(95,4 \%) 630 \mathrm{AC}$ & MTC-14027 & M & A & Diente \\
\hline$S$ & Los Molinos & $383 \pm 58$ & $1440 \mathrm{AD}(95,4 \%) 1690 \mathrm{AD}$ & MTC-14025 & $M$ & A & Diente \\
\hline$S$ & Nunsacat & $387 \pm 41$ & $1480 \mathrm{AD}(95,4 \%) 1650 \mathrm{AD}$ & MTC-13250 & $\mathrm{F}$ & AMD & Hueso \\
\hline$S$ & Los Molinos & $420 \pm 41$ & $1440 \mathrm{AD}(95,4 \%) 1620 \mathrm{AD}$ & MTC-13254 & M & AJ & Hueso \\
\hline $\mathrm{S}$ & Charquina & $445 \pm 38$ & $1420 \mathrm{AD}(95,4 \%) 1590 \mathrm{AD}$ & MTC-13253 & $\mathrm{F}$ & $\mathrm{AM}$ & Diente \\
\hline $\mathrm{S}$ & Ischilin & $459 \pm 40$ & $1310 \mathrm{AD}(95,4 \%) 1480 \mathrm{AD}$ & MTC-13256 & M & AMD & Hueso \\
\hline$S$ & $\begin{array}{l}\text { Sta Rosa de } \\
\text { Calamuchita }\end{array}$ & $533 \pm 42$ & $1330 \mathrm{AD}(95,4 \%) 1510 \mathrm{AD}$ & AA102659 & M & AMD & Diente \\
\hline $\mathrm{S}$ & Constantinopla & $619 \pm 43$ & $1240 \mathrm{AD}(95,4 \%) 1420 \mathrm{AD}$ & $\mathrm{AA}_{102658}$ & M & AMD & Hueso \\
\hline$S$ & Cerro Colorado & $664 \pm 33$ & $1210 \mathrm{AD}(95,4 \%) 1360 \mathrm{AD}$ & MTC-13216 & $\mathrm{F}$ & A & Diente \\
\hline$S$ & Copina & $680 \pm 40$ & $1180 \mathrm{AD}(95,4 \%) 1350 \mathrm{AD}$ & MTC-13248 & M & A & Diente \\
\hline
\end{tabular}




\begin{tabular}{|c|c|c|c|c|c|c|c|}
\hline Región & Sitio & $\begin{array}{c}\text { Fechados }{ }^{14} \mathrm{C} \\
\text { (años AP) }\end{array}$ & $\begin{array}{c}\text { Fechados calibrados } 2 \\
\text { sigmas }\end{array}$ & $\begin{array}{l}\text { Código de } \\
\text { laboratorio }\end{array}$ & Sexo & Edad & $\begin{array}{l}\text { Material } \\
\text { fechado }\end{array}$ \\
\hline S & $\begin{array}{l}\text { Banda Mnal del } \\
\text { Lago }\end{array}$ & $695 \pm 20$ & $1210 \mathrm{AD}(95,4 \%) 1295 \mathrm{AD}$ & $\mathrm{UCl} 39104$ & M & AJ & Hueso \\
\hline$S$ & Rosca Yaco & $705 \pm 131$ & $950 \mathrm{AD}(95,4 \%) 1550 \mathrm{AD}$ & MTC-13252 & M & A & Hueso \\
\hline$S$ & Los Molinos & $881 \pm 150$ & $750 \mathrm{AD}(95,4 \%) 1400 \mathrm{AD}$ & MTC-13215 & M & A & Diente \\
\hline$S$ & Los Molinos & $889 \pm 59$ & $940 \mathrm{AD}(95,4 \%) 1180 \mathrm{AD}$ & MTC-14028 & $\mathrm{F}$ & AMD & Diente \\
\hline$S$ & Guasmara & $920 \pm 20$ & $985 \mathrm{AD}(95,4 \%) 1070 \mathrm{AD}$ & $\mathrm{UCl} 22281$ & M & A & Hueso \\
\hline$S$ & Loma Bola & $954 \pm 85$ & $820 \mathrm{AD}(95,4 \%) 1170 \mathrm{AD}$ & MTC-128o6 & $\mathrm{F}$ & A & Diente \\
\hline$S$ & San Esteban & $965 \pm 15$ & $950 \mathrm{AD}(95,4 \%) 1015 \mathrm{AD}$ & $\mathrm{UCl}_{39103}$ & $\mathrm{~F}$ & AJ & Hueso \\
\hline$S$ & Rio III-Quillinzo & $975 \pm 38$ & $890 \mathrm{AD}(95,4 \%) 1060 \mathrm{AD}$ & MTC-13245 & M & AJ & Diente \\
\hline$S$ & Los Molinos & $981 \pm 41$ & $880 \mathrm{AD}(95,4 \%)$ 106o AD & MTC-13251 & M & A & Diente \\
\hline$S$ & Los Molinos & $995 \pm 161$ & $600 \mathrm{AD}(95,4 \%) 1300 \mathrm{AD}$ & MTC-13246 & M & AM & Diente \\
\hline$S$ & Guasmara & $\mathrm{S} / \mathrm{F}$ & - & - & $\mathrm{F}$ & AMD & - \\
\hline$S$ & Loma Bola & $\mathrm{S} / \mathrm{F}$ & - & - & $M$ & AMD & Diente \\
\hline$S$ & Los Molinos & $\mathrm{S} / \mathrm{F}$ & - & - & $M$ & AMD & - \\
\hline$S$ & Los Molinos & $\mathrm{S} / \mathrm{F}$ & - & - & M & A & - \\
\hline$S$ & Nunsacat & $S / F$ & - & - & M & AMD & - \\
\hline$S$ & Potrero de Garay & $S / F$ & - & - & $\mathrm{F}$ & AJ & - \\
\hline $\mathrm{S}$ & Potrero de Garay & $\mathrm{S} / \mathrm{F}$ & - & - & M & AMD & - \\
\hline$S$ & $\begin{array}{l}\text { Santa Rosa de } \\
\text { Calamuchita }\end{array}$ & $\mathrm{S} / \mathrm{F}$ & - & - & $M$ & AJ & - \\
\hline
\end{tabular}

Tabla 1. Muestras analizadas. Referencias: S (Sierras), LL (Llanuras); Código del Laboratorio: MTC (Graduate School of Frontier Sciences, University of Tokio), UCl (Earth System Science Dept., University of Irving), AA (University of Arizona Accelerator Mass Spectrometry Laboratory); Sexo: F (Femenino), M (Masculino); Edad: A (adulto), AJ (Adulto joven), MD (adulto medio), AM (adulto mayor). Los fechados radiocarbónicos fueron calibrados con el programa OxCal v3.10, Bronk Ramsey (2005), se presentan los resultados de la calibración con 2 sigmas/probabilidad de un 95,4\%.

de los alimentos más que cambios a nivel dietario hacia momentos finales del Holoceno tardío (Fabra y González, 2016; Laguens, Fabra, Macedo do Santos y Demarchi, 2009).

Por otro lado, la evidencia bioantropológica nos informa sobre la existencia de diferencias -morfológicas y genéticas- entre las poblaciones asentadas en las regiones Sierras y Llanuras a partir de los 1200 años AP. Los cambios en ciertas estructuras de la morfología craneofacial estarían sugiriendo diferencias significativas entre las poblaciones del Holoceno medio y del Holoceno tardío. También se registraron diferencias entre las poblaciones asentadas en las Llanuras y aquellas de las Sierras, registrándose mayores similitudes morfológicas para las Sierras con poblaciones de la región Chaco-Santiagueña y del oeste de la región Pampeana, mientras que en las Llanuras las similitudes estarían dadas con poblaciones del noreste de Patagonia (Fabra y Demarchi, 2013). Por otro lado, estudios realizados por Nores, Fabra y Demarchi (2011) han estimado la existencia de diferencias estadísticamente significativas en la distribución haplotípica entre los habitantes de las Sierras y de las Llanuras de Córdoba las cuales se habrían generado a partir de 1200 años AP. Estas diferencias se manifiestan en la distribución de frecuencias de los haplogrupos mitocondriales, tales como elevada frecuencia de haplogrupo B en los individuos de las Sierras y de los haplogrupos A y D en la Llanura. A partir de estas diferencias los autores sugieren que alrededor de los 1200 años AP se habrían producido contactos o migraciones desde el Noreste y de la región Chaqueña con las poblaciones asentadas en Sierras, y desde el Noreste y Este de nuestro país hacia la región Llanuras (Fabra y Demarchi, 2013; Nores y Demarchi, 2011; Nores et al., 2011). 
Los análisis bioantropológicos se realizaron siguiendo los procedimientos establecidos por Buikstra y Ubelaker (1994). Para la determinación del sexo se tuvieron en cuenta las características de la pelvis (región subpúbica, escotadura ciática y surco preauricular), complementándola con rasgos de la morfología craneal (desarrollo de la cresta nucal, tamaño del proceso mastoideo, prominencia de la glabela y proyección de la eminencia mental). Para la estimación de la edad se consideraron los cambios en la sínfisis púbica (Gilbert y Mckern, 1973; Todd, 1921), la superficie auricular del ilion (Lovejoy, Meindl, Mensforth y Barton, 1985) y la morfología esternal de la cuarta costilla, según la propuesta de Krenzer (2006). En los casos en que disponíamos sólo del cráneo se consideró el método de la obliteración de las suturas ectocraneales (Meindl y Lovejoy, 1985).

Para el registro del desgaste dental se decidió aplicar la escala ordinal de ocho grados propuesta por Smith (1984), la cual considera tanto dientes anteriores -incisivos, caninos y premolares- como posteriores - molares-. Para el registro de forma y patrón del desgaste dental se siguió la propuesta metodológica Molnar (1971), con algunas simplificaciones de las variables. En este sentido, se consideraron sólo siete categorías de la variable forma y patrón de desgaste -desgaste plano, oblicuo hacia bucal, oblicuo hacia lingual, cóncavo, redondeado y surcos.

Se decidió evaluar la prevalencia de los bioindicadores por individuo y para el total de la muestra, considerando su distribución según diferentes categorías: sexo (femeninos $\mathrm{n}=19$, masculinos $\mathrm{n}=42$ ), edad (adultos jóvenes $-20-34$ años $-\mathrm{n}=10$, medios $-35-50$ años- $\mathrm{n}=20$, adultos mayores - más de 50 años- $\mathrm{n}=5$, y adultos -individuos que no se han podido adscribir a ningún rango etario- $\mathrm{n}=26$ ), regiones (Sierras y Llanuras) y cronología (pre 1200 años AP y post 1200 AP). Al trabajar con variables cualitativas ordinales, en el caso del grado de desgaste, se decidió aplicar el test de chi cuadrado para evaluar la significancia estadística de las diferencias observadas.

\section{Resultados}

Toda la muestra presenta un grado de desgaste de moderado a elevado. Más de la mitad de los individuos presentan exposición de dentina moderada. En la Tabla 2 se presentan las prevalencias de los distintos grados de desgaste para toda la muestra, discriminados por tipo de dientes. Como se puede observar, el grado de desgaste 6 según la escala de Smith (1984) es el más representado $(21,84 \%)$, mientras que el grado 5 es el segundo más representado en la muestra (19,78\%). Esto nos permite sugerir que más de la mitad de la muestra presenta exposición de dentina moderada a grave (grados 5, 6, 7, 8) en un $74,53 \%(761 / 1.021)$ y el resto presenta un $25,46 \%(260 / 1.021)$ de poco desgaste a moderado (grados 1, 2, 3, 4).

Los grados más avanzados de desgaste afectan en proporciones similares a toda la dentición, siendo el diente que presenta los mayores grados de desgaste el primer molar, aunque en general, a partir de los grados 5, 6, 7 y 8 todas las piezas dentales son afectadas en forma regular (Tabla 2). Se pudo observar que los primeros molares del maxilar inferior presentan mayor grado de desgaste que sus antagonistas del maxilar superior registrándose grados de desgaste entre 7 y 8 .

En cuanto al patrón y forma del desgaste dental observamos que el desgaste plano es el más preponderante en la muestra $(41,23 \%)$, seguido por el desgaste cóncavo $(24,48 \%)$, el desgaste oblicuo hacia lingual (14,48\%), el desgaste oblicuo hacia bucal $(11,45 \%)$ y el desgaste redondeado (7,24\%). En cuanto a las piezas dentales, el desgaste plano afecta en mayor medida a los terceros molares (57\%) junto a los segundos incisivos $(55,14 \%)$, siendo el primer molar el menos afectado por esta forma de desgaste (15,03\%). El patrón de desgaste tipo cóncavo se encuentra presente en mayor medida en el primer $(59,39 \%)$ 


\begin{tabular}{|c|c|c|c|c|c|c|c|c|c|c|c|c|c|c|c|c|c|}
\hline Grado & & 1 & & 2 & & 3 & & 4 & & 5 & & 6 & & 7 & & 8 & \\
\hline Diente & $\mathbf{N}$ & $\%$ & $\mathbf{N}$ & $\%$ & $\mathbf{N}$ & $\%$ & $\mathbf{N}$ & $\%$ & $N$ & $\%$ & $\mathbf{N}$ & $\%$ & $\mathbf{N}$ & $\%$ & $\mathbf{N}$ & $\%$ & Thear \\
\hline$M_{3}$ & 13 & 12,74 & 11 & 10,75 & 12 & 11,76 & 21 & 20,58 & 14 & 13,72 & 16 & 15,68 & 9 & 8,82 & 3 & 2,94 & 102 \\
\hline $\mathrm{M} 2$ & 2 & 1,42 & 7 & 5 & 5 & 3,57 & 13 & 9,28 & 18 & 12,85 & 31 & 22,14 & 38 & 27,14 & 15 & 10,71 & 140 \\
\hline $\mathrm{M}_{1}$ & 0 & 0 & 2 & 1,5 & 4 & 3 & 4 & 3 & 10 & 7,51 & 33 & 24,81 & 50 & 37,59 & 30 & 22,55 & 133 \\
\hline $\mathrm{PM}_{2}$ & 0 & o & 9 & 5,96 & 13 & 8,6 & 15 & 9,93 & 30 & 19,86 & 37 & 24,5 & 21 & 13,9 & 26 & 17,21 & 151 \\
\hline $\mathrm{PM}_{1}$ & 1 & 0,66 & 11 & 7,33 & 9 & 6 & 14 & 9,33 & 36 & 24 & 31 & 20,66 & 20 & 13,33 & 28 & 18,66 & 150 \\
\hline C & 3 & 2 & 8 & 5,33 & 6 & 4 & 18 & 12 & 46 & 30,66 & 27 & 18 & 19 & 12,66 & 23 & 15,33 & 150 \\
\hline 12 & 4 & 3,8 & 3 & 2,85 & 2 & 1,9 & 20 & 19,04 & 24 & 22,85 & 26 & 24,71 & 9 & 8,57 & 16 & 15,23 & 105 \\
\hline $\mathrm{I}_{1}$ & 2 & 2,22 & 3 & 3,33 & 2 & 2,22 & 16 & 17,77 & 24 & 26,66 & 22 & 24,44 & 10 & 11,11 & 12 & 13,33 & 90 \\
\hline Total & 25 & 2,44 & 54 & 5,28 & 53 & 5,19 & 121 & 11,85 & 202 & 19,78 & 223 & 21,84 & 176 & 17,23 & 153 & 14,98 & 1021 \\
\hline
\end{tabular}

Tabla 2. Prevalencias generales de grado de desgaste dental para el total de la muestra analizada. Referencias: M3: Tercer Molar, M2: Segundo Molar, M1: Primer Molar, PM2: Premolar segundo, PM1: Premolar Primero, C: Canino, I2: Incisivo Segundo, l1: Incisivo Primero: N: Número de dientes con desgaste: 1, 2, 3, 4, 5, 6, 7, 8: grados de desgaste dental.

y segundo molar (49,28\%). Por otra parte, el desgaste oblicuo hacia lingual afecta a los segundos incisivos $(21,11 \%)$ y segundos premolares (18\%). El desgaste oblicuo hacia bucal es mas preponderante en la dentición anterior, siendo los incisivos primeros (21,11\%), los segundos premolares (18\%), los segundos incisivos $(16,89 \%)$ y los caninos $(17,64 \%)$ los más afectados, al tiempo que los primeros $(7,85 \%)$ y segundos molares (7,51\%) presentan menor prevalencia de este patrón de desgaste, de acuerdo a lo esperado. El desgaste redondeado también presenta mayor incidencia en los dientes anteriores, incisivos primeros $(13,33 \%)$ e incisivos laterales $(13,08 \%)$, disminuyendo a casi la mitad en los dientes posteriores. Por último, la presencia de surcos es escasa en la muestra $(0,88 \%)$ y afecta a los primeros incisivos (2,22\%) y caninos (1,96\%) (Tabla 3) (González, 2016).

\section{Grado de desgaste}

\section{Prevalencias por región}

Los individuos masculinos presentan mayores grados de desgaste que sus pares femeninos, tanto en Sierras como en Llanuras. En la Tabla 4 se observa que los individuos masculinos de Sierras presentan elevadas prevalencias de desgaste leves (grados 1,2,3), mientras que aquellos hallados en Llanuras los grados de desgaste son más graves (grados 5, 6, 7,8 ). Los individuos femeninos presentan similares prevalencias de desgaste en ambas regiones, con representatividad moderada de todos los grados de desgaste, sin embargo, se observa que la pérdida de altura coronal presenta mayores prevalencias en Llanuras (Tabla 4), siendo esta diferencia estadísticamente significativa (Tabla 5).

Cuando observamos la distribución del desgaste en las distintas categorías etarias y su distribución geográfica, registramos que en Llanuras los adultos, adultos medios y mayores presentan mayores frecuencias de desgastes avanzados. En cambio, los adultos jóvenes presentan los desgastes más moderados. En Sierras el patrón es similar: los individuos adultos mayores y medios son los que presentan los desgastes más graves (grados 7 y 8 ), en estos últimos no se registran piezas dentales con menores grados de desgaste, lo que indica que el desgaste se incrementa notoriamente a medida que se avanza con la edad (Figura 2A).

\section{Prevalencias por cronología}

Al evaluar los grados de desgaste considerando dos períodos cronológicos, se registra que en momentos previos a los 1200 años AP, tanto individuos femeninos como masculinos 


\begin{tabular}{|c|c|c|c|c|c|c|c|c|c|c|c|c|c|}
\hline \multirow{2}{*}{$\begin{array}{l}\text { Patrón } \\
\text { Diente }\end{array}$} & \multicolumn{2}{|c|}{1} & \multicolumn{2}{|c|}{2} & \multicolumn{2}{|c|}{3} & \multicolumn{2}{|c|}{4} & \multicolumn{2}{|c|}{5} & \multicolumn{2}{|c|}{6} & \multirow{2}{*}{ Total } \\
\hline & $\mathbf{N}$ & $\%$ & $N$ & $\%$ & $N$ & $\%$ & $N$ & $\%$ & $N$ & $\%$ & $\mathbf{N}$ & $\%$ & \\
\hline$M_{3}$ & 57 & 57 & 11 & 11 & 8 & 8 & 23 & 23 & 1 & 1 & 0 & o & 100 \\
\hline $\mathrm{M} 2$ & 39 & 28,05 & 11 & 7,85 & 19 & 13,63 & 69 & 49,64 & 1 & 0,71 & 1 & 0,71 & 139 \\
\hline $\mathrm{M}_{1}$ & 21 & 15,03 & 10 & 7,51 & 14 & 10,52 & 78 & 58,64 & 9 & 6,76 & 1 & 0,75 & 133 \\
\hline $\mathrm{PM}_{2}$ & 63 & 42 & 23 & 15,33 & 27 & 18 & 27 & 18 & 10 & 6,66 & 0 & o & 150 \\
\hline $\mathrm{PM}_{1}$ & 68 & 45,27 & 15 & 10,13 & 25 & 16,89 & 29 & 19,59 & 12 & 8,1 & 0 & o & 149 \\
\hline C & 68 & 44,44 & 21 & 13,72 & 27 & 17,64 & 19 & 12,41 & 15 & 9,8 & 3 & 1,96 & 153 \\
\hline 12 & 59 & 55,14 & 15 & 14,01 & 12 & 11,21 & 5 & 4,67 & 14 & 13,08 & 2 & 1,86 & 107 \\
\hline$I_{1}$ & 46 & 51,11 & 10 & 11,11 & 19 & 21,11 & 1 & 1,11 & 12 & 13,33 & 2 & 2,22 & 90 \\
\hline Total & 421 & 41,23 & 116 & 11,45 & 151 & 14,48 & 250 & 24,48 & 74 & 7,24 & 9 & 0,88 & 1021 \\
\hline
\end{tabular}

Tabla 3. Cantidad y prevalencia de piezas dentales disponibles para el registro de patrón desgaste dental discriminadas por tipo de dientes. Referencias: M3: tercer molar, M2: segundo molar, M1: primer molar, PM2: segundo premolar, PM1: primer premolar, C: canino, I2: incisivo segundo, 11: incisivo primero, 1: Desgaste Plano, 2: Oblicuo hacia bucal, 3: Oblicuo hacia lingual, 4: Cóncavo, 5: Redondeado, 6: Surcos oclusales.

\begin{tabular}{|c|c|c|c|c|c|c|c|c|}
\hline \multirow[b]{3}{*}{ Grados } & \multicolumn{4}{|c|}{ Sierras } & \multicolumn{4}{|c|}{ Llanuras } \\
\hline & \multicolumn{2}{|c|}{ Femenino } & \multicolumn{2}{|c|}{ Masculino } & \multicolumn{2}{|c|}{ Femenino } & \multicolumn{2}{|c|}{ Masculino } \\
\hline & $\mathrm{N}$ & $\%$ & $N$ & $\%$ & $\mathrm{~N}$ & $\%$ & $\mathbf{N}$ & $\%$ \\
\hline 1 & 5 & 2,65 & 16 & 3,92 & 3 & 2,38 & 1 & 0,33 \\
\hline 2 & 8 & 4,25 & 35 & 8,51 & 8 & 6,34 & 3 & 1,0 \\
\hline 3 & 7 & 3,72 & 38 & 9,31 & 3 & 2,38 & 5 & 1,67 \\
\hline 4 & 32 & 17,02 & 68 & 16,66 & 6 & 4,76 & 21 & 7,02 \\
\hline 5 & 54 & 28,72 & 59 & 14,46 & 36 & 28,57 & 57 & 19,06 \\
\hline 6 & 39 & 20,74 & 91 & 22,30 & 24 & 19,04 & 69 & 23,07 \\
\hline 7 & 24 & 12,76 & 70 & 17,15 & 14 & 11,11 & 71 & 23,74 \\
\hline 8 & 19 & 10,10 & 31 & 7,59 & 32 & 25,39 & 72 & 24,08 \\
\hline Total & 188 & & 408 & & 126 & & 299 & \\
\hline
\end{tabular}

Tabla 4. Prevalencias generales de grado de desgaste discriminadas por sexo y subregión. Se muestran las prevalencias de los distintos grados de desgaste observados sin considerar tipos de dientes. Referencias: N: Número de piezas dentales afectadas 1, 2, 3, 4, 5, 6, 7, 8: grados de desgaste dental, \%: porcentaje de dientes afectados por los distintos grados de desgaste.

presentan mayor frecuencia de desgastes avanzados (grados 5, 6, 7 y 8), siendo los femeninos quienes presentan mayor prevalencia del grado más elevado de desgaste (Tabla 6). En momentos posteriores a los 1200 años AP se registra un aumento estadísticamente significativo en las frecuencias de todos los grados de desgaste (Tabla 5), registrándose en los individuos femeninos una mayor incidencia de desgaste moderado y en los individuos masculinos una mayor incidencia de desgaste grave (Tabla 6).

Cuando consideramos el desgaste dental según el tipo de diente afectado, vemos que en momentos anteriores a los 1200 años AP los individuos masculinos presentan mayor grado de desgaste en los primeros molares, con grados avanzados (56,6\%). De la misma manera, los individuos femeninos presentan desgaste en grados elevados (grados 7 y 8 ) en los primeros y segundos molares. También se observa que los individuos femeninos presentan mayor desgaste en los primeros premolares (50\%) e incisivos primeros $(30,76 \%)$.

Para momentos posteriores a los 1200 años AP la distribución del desgaste es más homogénea en todos los tipos de dientes y se observa una mayor frecuencia de todos 


\begin{tabular}{|l|c|c|c|c|}
\hline Grado de desgaste avanzado & $\begin{array}{c}\text { Ausencia/ } \\
\text { presencia }\end{array}$ & $\mathbf{X}^{\mathbf{2}}$ & Prob. & gl \\
\hline Por cronología & $0 / 18$ & & & \\
Pre 1200 AP & $12 / 21$ & & & \\
Post 1200 AP & $12 / 49$ & 4,612 & 0,032 & 1 \\
Total & $1 / 27$ & & & \\
\hline Por región & $11 / 22$ & & & \\
Llanuras & $22 / 47$ & 5,590 & 0,018 & \\
Sierras & $4 / 6$ & & & \\
Total & $1 / 20$ & & & \\
\hline Por edad & $0 / 6$ & & & \\
Adulto joven & $7 / 17$ & & & \\
Adulto medio & $12 / 49$ & 10,015 & 0,018 & \\
Adulto mayor & & & & \\
Adulto & & & & \\
\hline
\end{tabular}

Tabla 5. Resultados del Test Chi-Cuadrado. Se presentan solo los resultados estadísticamente significativos. "Se redujeron las presencias de grados de desgaste entre leves (grados 1 a 4 ) y avanzado (5 a8).

los grados de desgaste. Particularmente para los individuos masculinos se registra una mayor afectación de la dentición posterior, en cambio en los femeninos los dientes más afectados son los de la dentición anterior: primer premolar $(48,14 \%)$, caninos $(52,94 \%)$ e incisivos (50\%) con grado de desgaste 5 .

En la Figura 2B se observan las diferencias entre las categorías etarias analizadas y los períodos cronológicos considerados. En el período anterior a los 1200 años AP los adultos medios y mayores presentan los mayores grados de desgaste llegando a extremos donde se pierde la funcionalidad de las piezas dentales y se exhiben restos radiculares, siendo los adultos jóvenes quienes presentan desgaste leve a moderado. En cambio, hacia momentos posteriores a los 1200 años AP, la variabilidad en los distintos grados de desgaste es mayor en todas las categorías etarias, aunque se observa que los jóvenes presentan menores grados de desgaste y los adultos medios y mayores presentan desgastes moderados a graves. En la totalidad de la muestra se registra una diferencia significativa de los grados de desgaste grave en los adultos medios (Tabla 5) mientras que los adultos mayores presentan todos desgaste avanzado, sin embargo, consideramos que otros indicadores como el aumento de las prevalencias de pérdidas dentales antemortem podrían también estar afectando el registro de los grados de desgaste, sumado esto al bajo número muestral para estas categorías etarias.

\section{Patrón y forma de desgaste}

\section{Prevalencias por región}

Se observa que el desgaste plano está presente en ambos sexos de la región Sierras en elevada frecuencia, siendo un poco más afectados los individuos masculinos (56,37\%). Es notable que en esta región los individuos femeninos presenten mayor prevalencia de los desgastes oblicuos que sus pares masculinos. Para la región Llanuras, las formas planas del desgaste disminuyen en promedio para ambos sexos en comparación con la región Sierras (Tabla 7). El desgaste cóncavo se presenta en prevalencias similares para ambos sexos. Se registra una diferencia en cuanto a la presencia de desgaste oblicuo hacia lingual, más frecuente en individuos femeninos (35,71\%). 


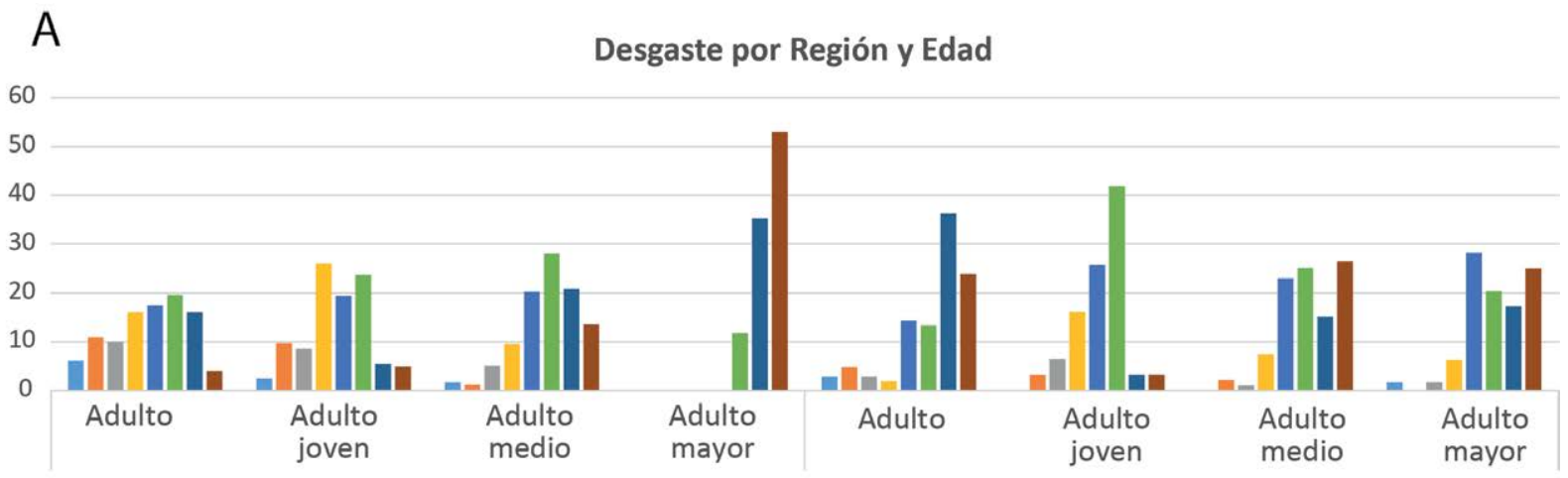

Sierras

Llanura

B

Edad - Pre 1200 años AP

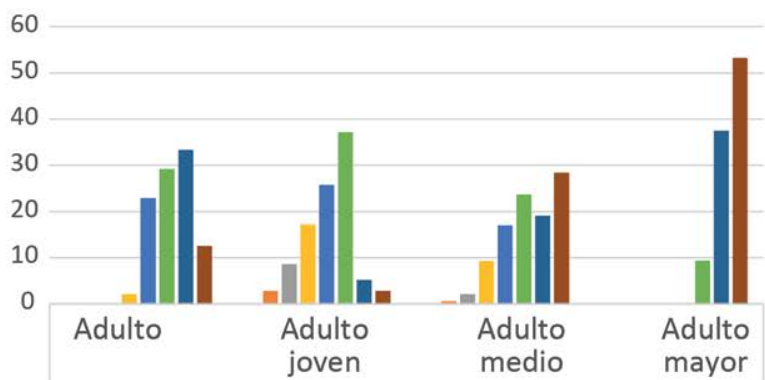

Edad - Post 1200 AP

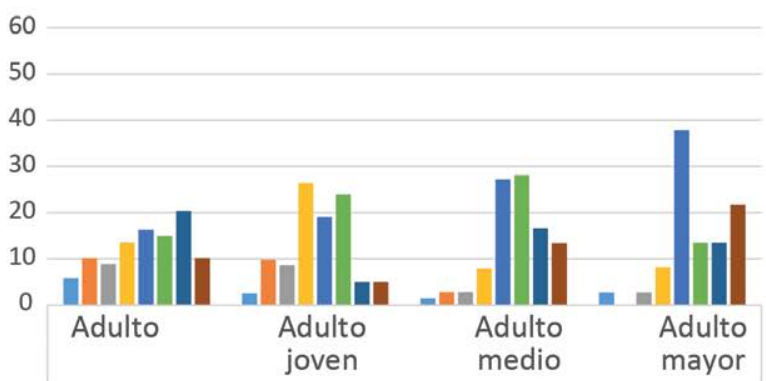

Figura 2. A) prevalencias generales de grado de desgaste en individuos femeninos y masculinos según la ubicación geográfica; B) prevalencias generales por grupos de edad discriminados por periodo cronológico (Referencias: 1, 2, 3, 4, 5, 6, 7, 8: grados de desgaste dental)

\begin{tabular}{|c|c|c|c|c|c|c|c|c|}
\hline \multirow[b]{3}{*}{ Grados } & \multicolumn{4}{|c|}{ Pre 1200 años AP } & \multicolumn{4}{|c|}{ Post 1200 años AP } \\
\hline & \multicolumn{2}{|c|}{ Femenino } & \multicolumn{2}{|c|}{ Masculino } & \multicolumn{2}{|c|}{ Femenino } & \multicolumn{2}{|c|}{ Masculino } \\
\hline & $\mathbf{N}$ & $\%$ & $\mathbf{N}$ & $\%$ & $\mathbf{N}$ & $\%$ & $\mathbf{N}$ & $\%$ \\
\hline 1 & 2 & 1,73 & $\mathrm{o}$ & o & 6 & 3,03 & 17 & 3,32 \\
\hline 2 & 1 & 0,86 & 2 & 1,02 & 15 & 7,57 & 36 & 7,04 \\
\hline 3 & 3 & 2,6 & 4 & 2,04 & 7 & 3,55 & 42 & 8,21 \\
\hline 4 & 12 & 10,41 & 14 & 7,14 & 26 & 13,13 & 78 & 15,26 \\
\hline 5 & 20 & 17,39 & 32 & 16,32 & 71 & 35,85 & 83 & 16,24 \\
\hline 6 & 20 & 17,39 & 54 & 27,55 & 40 & 20,2 & 106 & 20,74 \\
\hline 7 & 20 & 17,39 & 46 & 23,46 & 17 & 8,58 & 88 & 17,22 \\
\hline 8 & 37 & 32,17 & 44 & 22,44 & 16 & 8,08 & 61 & 11,93 \\
\hline Total & 115 & & 196 & & 198 & & 511 & \\
\hline
\end{tabular}

Tabla 6. Prevalencias generales de grado de desgaste en individuos femeninos y masculinos según los periodos cronológicos. Referencias: N: cantidad de piezas dentales afectadas; 1, 2, 3, 4, 5, 6, 7, 8: grados de desgaste dental.

En cuanto a los patrones de desgaste dental considerando los tipos de dientes, observamos que el desgaste plano sigue predominando en toda la muestra. Sin embargo, se puede observar que en individuos femeninos y masculinos de la región Sierras está presente en mayor prevalencia en todas las piezas dentales, disminuyendo en los primeros molares en donde el desgaste cóncavo es mayor. En la región Llanuras la 


\begin{tabular}{|c|c|c|c|c|c|c|c|c|}
\hline \multirow[b]{3}{*}{ Patrón } & \multicolumn{4}{|c|}{ Sierras } & \multicolumn{4}{|c|}{ Llanuras } \\
\hline & \multicolumn{2}{|c|}{ Femeninos } & \multicolumn{2}{|c|}{ Masculinos } & \multicolumn{2}{|c|}{ Femeninos } & \multicolumn{2}{|c|}{ Masculinos } \\
\hline & $N$ & $\%$ & $N$ & $\%$ & $N$ & $\%$ & $\mathbf{N}$ & $\%$ \\
\hline 1 & 84 & 44,68 & 230 & 56,37 & 28 & 22,22 & 77 & 25,75 \\
\hline 2 & 23 & 12,23 & 24 & 5,88 & 10 & 7,93 & 59 & 19,73 \\
\hline 3 & 27 & 14,36 & 33 & 8,08 & 45 & 35,71 & 46 & 15,38 \\
\hline 4 & 43 & 22,87 & 102 & 25 & 30 & 23,8 & 75 & 25,08 \\
\hline 5 & 11 & 5,85 & 14 & 3,43 & 12 & 9,52 & 39 & 13,14 \\
\hline 6 & $\mathrm{o}$ & o & 5 & 1,22 & 1 & 0,79 & 3 & 1 \\
\hline Total & 188 & & 408 & & 126 & & 299 & 1021 \\
\hline
\end{tabular}

Tabla 7. Prevalencia de patrones de desgaste dental discriminadas por sexo y región. Referencias: N: Número de piezas dentales afectadas, \%: prevalencia, 1: Desgaste Plano, 2: Oblicuo hacia bucal, 3: Oblicuo hacia lingual, 4: Cóncavo, 5: Redondeado, 6: Surcos oclusales, Total: Suma de piezas dentales.

variabilidad en cuanto a las formas de los desgastes dentales es mayor: en femeninos predominan los oblicuos hacia linguales en primeros molares (40\%), segundos premolares $(38,09 \%)$, primeros premolares $(42,1 \%)$ e incisivos. En masculinos los patrones de desgastes redondeados afectan a los premolares $(27,27 \%)$ mientras que las formas cóncavas afectan mayormente a los molares $(53,13 \%)$.

Por último, evaluamos la distribución de estos patrones de desgaste considerando los distintos grupos etarios por regiones (Figura 3A). En la región Llanuras las formas de desgaste son más variadas, en las categorías adultos y adultos jóvenes el desgaste oblicuo hacia lingual es el más frecuente $(38,7 \%)$, mientras que en adultos medios el desgaste cóncavo $(30,63 \%)$ predomina sobre las demás formas. En adultos mayores el desgaste plano $(23,07 \%)$, cóncavo $(25 \%)$ y redondeado $(23,07 \%)$ tiene prevalencias similares y moderadas. Es de destacar que en esta región la presencia de surcos se evidencia en adultos mayores $(3,84 \%)$. En la región Sierras el desgaste predominante es el plano en adultos jóvenes $(73,8 \%)$, adultos $(56,17 \%)$ y adultos medios $(33,52 \%)$. En general se registran menores prevalencias de las otras formas de desgaste en comparación con la región Llanuras (Figura 3A).

\section{Prevalencias por cronología}

Al evaluar si había diferencias temporales respecto a la presencia de los distintos patrones de desgaste dental, se observa que los individuos femeninos del período anterior a los 1200 años AP presentan mayor prevalencia de desgaste oblicuo hacia lingual (47,32\%), mientras que en los masculinos es mayor el desgaste cóncavo (30,96\%) y plano $(29,44 \%)$. Es de destacar que todos los surcos observados se registran en los individuos masculinos (Tabla 8). En momentos posteriores a 1200 años AP, las prevalencias de todos los patrones de desgaste se asemejan entre los sexos, siendo elevadas para el desgaste plano y cóncavo. En este período los individuos masculinos presentan elevada prevalencia de surcos oclusales.

En cuanto a los tipos de dientes, en momentos previos a 1200 años AP el desgaste plano afecta en individuos femeninos a los incisivos segundos (79,16\%), incisivos primeros $(61,11 \%)$ y terceros molares $(65,21 \%)$. Otro de los patrones más frecuentes es el de forma cóncava, que predomina en los primeros $(61,6 \%)$ y segundos molares $(42,3 \%)$. En masculinos, el desgate plano afecta incisivos $(42,85 \%)$ y premolares $(34,48 \%)$, y también los terceros molares presentan elevadas prevalencias de desgaste plano $(35,71 \%)$. El desgaste oblicuo hacia bucal se presenta con moderadas prevalencias en 


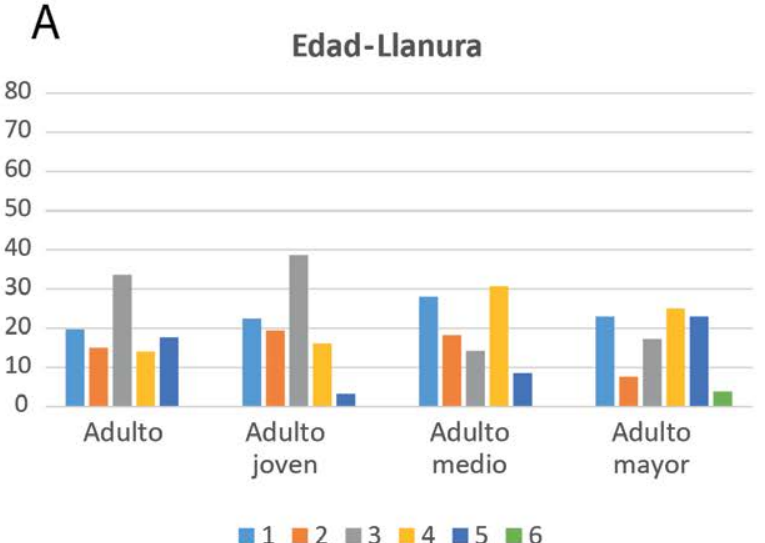

B

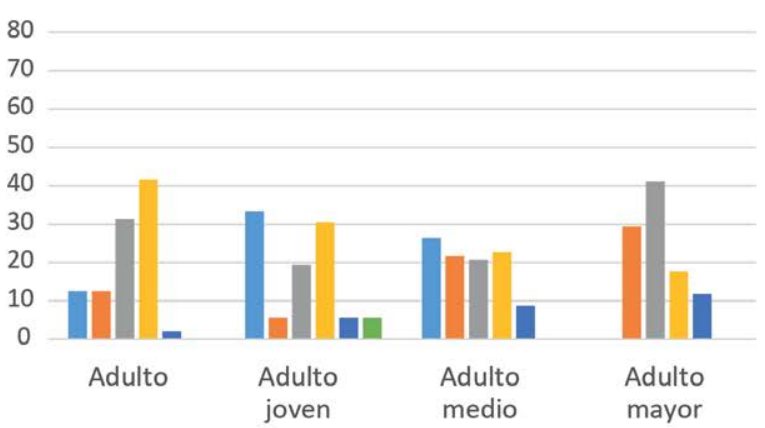

Edad-Sierras

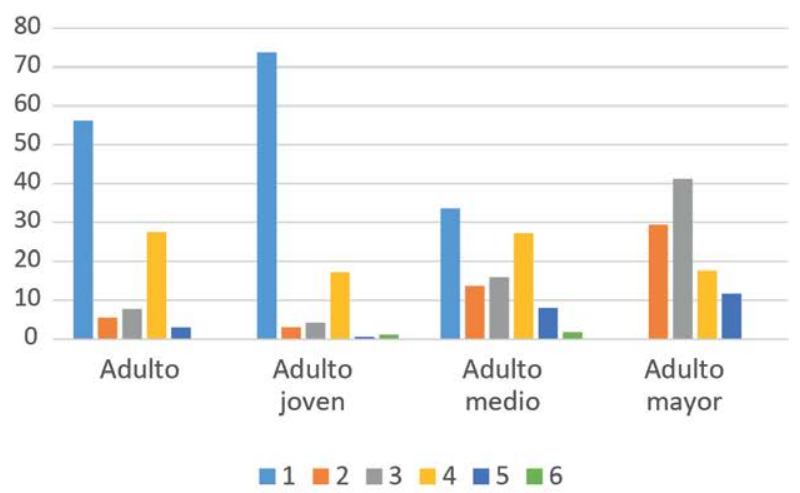

Edad- Post 1200 Años AP

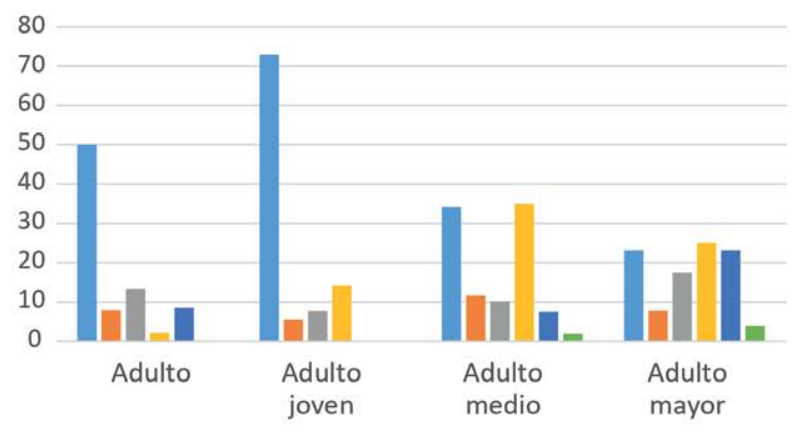

Figura 3. A) prevalencias generales discriminadas por categorías etarias y región. Se muestran las prevalencias de los distintos patrones de desgaste observados sin considerar tipos de dientes; $B$ ) prevalencias de los distintos patrones de desgaste observados sin considerar tipos de dientes, discriminadas por categorías etarias y cronología (Referencias: N: Número de piezas dentales afectadas, 2: Oblicuo hacia bucal, 3: Oblicuo hacia lingual, 4: Cóncavo, 5: Redondeado, 6: Surcos oclusales, \%: porcentaje de dientes afectados).

\begin{tabular}{|l|c|c|c|c|c|c|c|c|}
\cline { 2 - 10 } \multicolumn{1}{c|}{} & \multicolumn{4}{c|}{ Pre 1200 años AP } & \multicolumn{4}{c|}{ Post 1200 años AP } \\
\cline { 2 - 10 } \multicolumn{1}{c|}{} & \multicolumn{2}{c|}{ Femenino } & \multicolumn{2}{c|}{ Masculino } & \multicolumn{2}{c|}{ Femenino } & \multicolumn{3}{c|}{ Masculino } \\
\hline Patrón & N & \% & N & \% & N & $\%$ & N & \% \\
\hline 1 & 11 & 9,82 & 58 & 29,44 & 102 & 50,49 & 250 & 49,01 \\
2 & 18 & 16,07 & 40 & 20,3 & 14 & 6,92 & 43 & 8,43 \\
3 & 53 & 47,32 & 20 & 10,15 & 19 & 9,4 & 59 & 11,53 \\
4 & 20 & 17,85 & 61 & 30,96 & 52 & 25,74 & 116 & 22,74 \\
5 & 10 & 8,92 & 14 & 7,1 & 14 & 6,92 & 38 & 7,45 \\
6 & 0 & 0 & 4 & 2,03 & 1 & 0,49 & 4 & 0,78 \\
\hline Total & 112 & & 197 & & 202 & & 510 & \\
\hline
\end{tabular}

Tabla 8. Cantidad y prevalencia de piezas dentales disponibles para el registro de patrón desgaste dental discriminadas por sexo y cronología. Referencias: N: Número de piezas dentales afectadas, \%: prevalencia, 1: Desgaste Plano, 2: Oblicuo hacia bucal, 3: Oblicuo hacia lingual, 4: Cóncavo, 5: Redondeado, 6: Surcos oclusales.

premolares, particularmente en el segundo $(27,58 \%)$ y el primer molar $(24,13 \%)$. El desgaste cóncavo es más frecuente en molares, mientras que las formas redondeadas afectan a la dentición anterior. Debe destacarse que los surcos oclusales se presentan en forma predominante en caninos $(7,69 \%)$. 


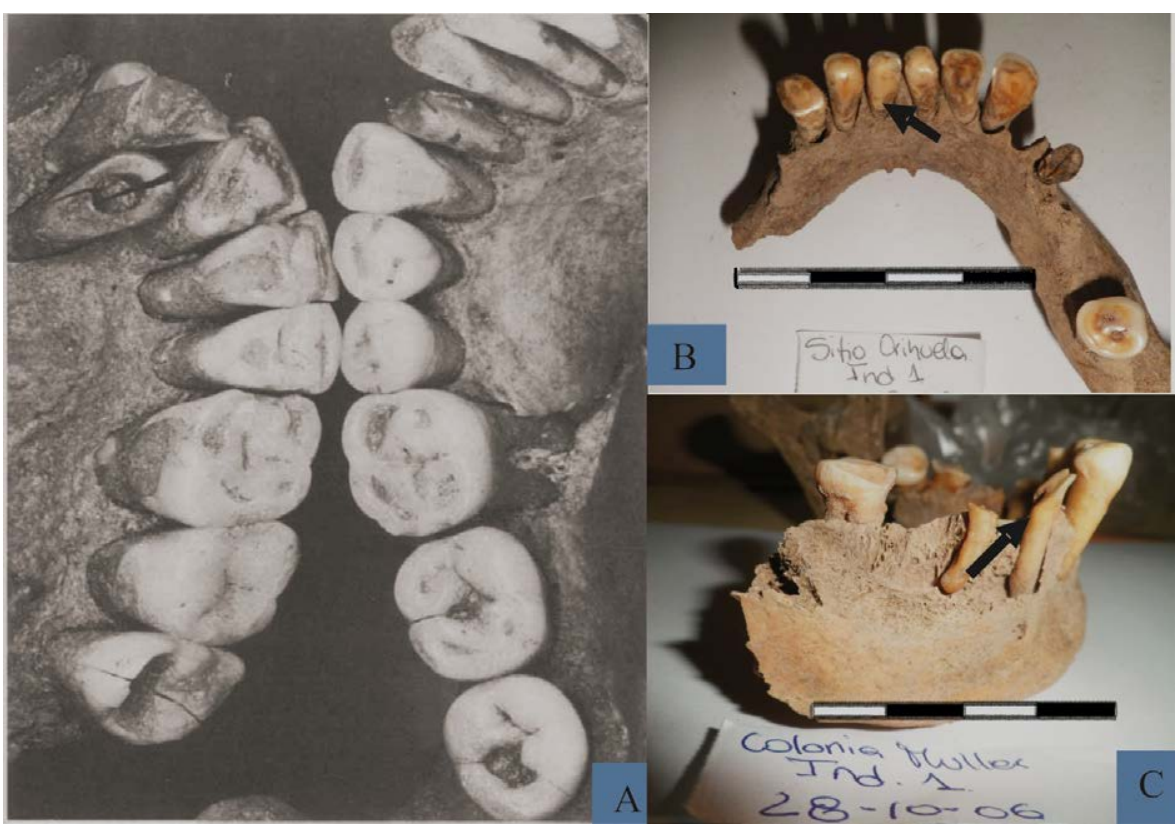

Figura 4. Patrones de desgaste. A: Imagen tomada del trabajo de Turner y Machado (1983) donde se observa un patrón de desgaste oblicuo hacia lingual predominante en maxilar superior. B: Maxilar inferior de un individuo femenino, obsérvese las pérdidas dentales premortem en molares derechos y la necrosis del segundo premolar derecho. Sitio Orihuela I1, región Llanuras; C: Maxilar inferior de individuo femenino; obsérvese desgaste oblicuo en premolares, este desgaste ha llegado a exponer la dentina llegando a la cavidad pulpar propiciando la presencia de caries. Sitio Colonia Muller l1, región Llanuras.

Para momentos posteriores a los 1200 años AP el desgaste plano continúa predominando en individuos masculinos en todos los tipos dentales, a excepción del primer molar en donde el patrón cóncavo es el más frecuente (64,38\%). Los desgastes oblicuos, tanto hacia bucal como lingual, presentan moderadas prevalencias en todos los dientes. Se registra que la forma redondeada afecta a incisivos primeros $(38,46 \%)$ mientras que los surcos se presentan en los incisivos laterales (4\%). En el caso de los individuos femeninos, los dientes más afectados son los premolares $(61,11 \%)$ e incisivos $(53,84 \%)$ con desgate oblicuo hacia lingual, el primer molar también es afectado por el desgaste oblicuo hacia lingual, pero las formas cóncavas y redondeadas también lo afectan en altas prevalencias.

En la figura 3B se presentan las prevalencias de los distintos patrones de desgaste dental, distribuidos por grupos etarios y por periodo cronológico. En momentos previos a los 1200 años AP, los patrones de desgaste que más inciden en adultos jóvenes son el plano (33,33\%), el cóncavo (30,35\%) y el oblicuo hacia lingual (19,34\%). La presencia de surcos sólo se registra en este grupo etario (5,55\%) debido posiblemente a que a mayor edad el desgaste dental elimina estos rasgos de la muestra. Los adultos medios presentan moderadas prevalencias de todos los patrones de desgaste, siendo el más elevado la forma plana $(26,28 \%)$, seguida por la forma cóncava $(22,68 \%)$ y las oblicuas hacia bucal $(21,26 \%)$ y lingual $(20,61 \%)$. En el caso de los adultos mayores, el patrón que predomina es el oblicuo hacia lingual $(41,17 \%)$, seguido en valores por el desgaste oblicuo hacia bucal $(29,41 \%)$ y en menor medida el cóncavo $(17,64 \%)$ y el redondeado (11,64\%). En el caso de los individuos incluidos en el grupo etario adultos, las formas que predominan son las cóncavas $(41,66 \%)$ y las oblicuas hacia lingual $(31,25 \%)$, las demás formas presentan moderadas a bajas prevalencias. Hacia finales del Holoceno tardío predominan las formas planas en los individuos adultos, adultos jóvenes y medios. En el caso de los adultos medio, el patrón preponderante es el cóncavo (35,02\%), al tiempo que una situación similar se observa en los adultos mayores (25\%), quienes presentan también prevalencias moderadas de las formas redondeadas $(23,07 \%)$. 


\section{Discusión}

Los resultados obtenidos en el presente estudio han permitido ahondar en el estudio de los grados y patrones de desgaste dental, los cuales serán vinculados con los tipos de alimentos consumidos y con las técnicas de procesamiento utilizadas para su cocción a lo largo del tiempo. En particular, hemos observado que las poblaciones asentadas tanto en la región serrana como en las llanuras orientales de la provincia presentan elevados grados de desgaste dental a lo largo de todo el Holoceno tardío y las formas planas son las que prevalecen en la muestra, sobre todo en Sierras. Las formas cóncavas también presentan prevalencias elevadas pero con valores similares en ambas regiones.

Si evaluamos en conjunto el avanzado grado de desgaste para toda la región y la predominancia de los patrones planos y cóncavos, podemos sugerir en primera instancia que serían el resultado de las altas exigencias masticatorias. En este caso no sólo debemos considerar los alimentos de origen vegetal, como es el caso de los frutos de algarroba ${ }^{3}$ ampliamente consumida en el centro de Argentina según fuentes etnohistóricas (Castro Olañeta, 2006), sino también los de origen cárnico. Si bien no conocemos formas de preparación y/o conservación de productos animales, estudios arqueológicos han sugerido el consumo de gran variedad de mamíferos y vertebrados de tamaño mediano a chico durante el Holoceno tardío (Rivero, Medina, Recalde y Pastor, 2010), así como una mayor inversión en el procesamiento y extracción de grasas y proteínas desde el Holoceno medio como parte de una estrategia de apropiación de recursos de amplio espectro. Consideramos que además de la ingesta de vegetales fibrosos y de estos pequeños mamíferos, el masticado de pequeños huesos de animales puede haber contribuido con el desgaste dental de estas poblaciones. Por otro lado consideramos que los patrones de desgaste registrados en la presente muestra para todo el Holoceno tardío se condicen con lo esperado para poblaciones con un modo de vida basado en una economía de tipo mixta, en donde la incorporación de la agricultura fue una estrategia más sumadas a la caza y la recolección, con una mayor importancia relativa en Sierras tal como lo indican los estudios bioantropológicos (Fabra et al., 2012a).

Se ha documentado que un cambio en la dieta y/o forma de preparación de los alimentos produce modificaciones sustanciales en el grado y la forma del desgaste dental (Deter, 2009; Larsen, 1985; Lev-Tov y Smith, 2006; Scott, 1979; Smith, 1984). En el caso de la muestra analizada en este trabajo, los cambios en el grado de desgaste son significativos entre ambas regiones y entre los dos momentos del Holoceno, afectando mayormente los primeros y segundos molares. Se observa que en la región Sierras el grado de desgaste disminuye hacia fines del Holoceno tardío y aumenta en la región Llanuras. Estas diferencias temporales y regionales podrían estar indicando la incorporación de nuevas técnicas de preparación de alimentos hacia fines del Holoceno tardío, sobre todo en Llanuras, antes que cambios sustanciales en el tipo de productos consumidos, sobre todo si consideramos lo que nos indican los valores de isótopos estables para estas poblaciones y las frecuencias de patologías orales (Fabra y González, 2016). La disminución del grado de desgaste registrado hacia momentos finales estaría indicando una modificación en los modos de preparación y cocción de los alimentos. La introducción de alimentos cultivados como el maíz, habría contribuido en forma relativa a esta disminución del estrés masticatorio, sobre todo en la región de Sierras, como lo sustentan el notable aumento en la prevalencia de caries hacia fines del Holoceno tardío (12,75\%) en comparación con lo registrado hacia momentos iniciales $(4,42 \%)$, sumado a datos isotópicos que nos señalan valores promedio de $\delta^{13} \mathrm{C}$ de $-13,8 \%$ para las poblaciones serranas para este momento (Fabra y González, 2015; González, 2016; González y Fabra, 2011). La disminución en los grados de desgaste dental ha sido registrado en las poblaciones cazadoras-recolectoras de Patagonia, Pampa y Cuyo sobre todo en momentos finales del Holoceno tardío e incluso en el período colonial, coincidentes con el aumento en las prevalencias de caries (Bernal et al., 2007; Menéndez, 2010).
3. Las chauchas de algarroba son caracterizadas como alimentos muy fibrosos, con elevadas cantidades de oxalatos y fitatos, lo que podría contribuir al desgaste dental (González Galán, Correa, Patto De Abreu y Piccolo Barcelos, 2008). 
4. En el sitio El Ranchito ubicado en la región Noroeste se han localizado grandes sitios con pozos subterráneos en forma de campana denominados comúnmente "botijas", en donde se han recuperado restos carbonizados de semillas, probablemente Prosopis sp. (Laguens y Bonnín, 1987). En sitios ubicados en la región Noreste también se han localizado concentraciones de estas estructuras de almacenamiento (Frenguelli y De Aparicio, 1932), en algunos casos asociadas a entierros humanos (Fabra et al., 2009).
Los individuos de la región Llanura presentan grados de desgaste más avanzados que los de las Sierras, con valores estadísticamente significativos, siendo los individuos masculinos los más afectados con patrones de desgaste variables en donde predominan las formas cóncavas y planas en ambos sexos, al tiempo que se destacan las formas oblicuas en femeninos y redondeadas en masculinos. Consideramos que estas diferencias entre las regiones hacen referencia a varios factores que estarían actuando en conjunto. Si los indicadores de patologías orales y los valores de isótopos estables que se cuentan para esta región nos indican que la dieta no tuvo cambios significativos a lo largo del Holoceno tardío, registrándose valores promedio de $-15 \%$ en $\delta^{13} \mathrm{C}$ (Fabra y González, 2016), podemos considerar que una dieta más abrasiva podría estar explicando estas diferencias y sobre todo las formas de cocción o preparación de los alimentos pueden haber influido en el avanzado desgaste dental registrado en las Llanuras, más allá de un cambio dietario sustancial. En esta región, y a diferencia de lo que ocurre en las Sierras, es muy común la presencia de grandes concentraciones de pozos subterráneos para el almacenamiento de frutos o recursos, denominados comúnmente como "hornillos o botijas" cuya función ha sido atribuida como almacenamiento o incluso en la preparación de alimentos (Cornero, Del Río y Ceruti, 2013; Fabra et al., 2009; Laguens y Bonnin, 2009). Es destacable la presencia de este tipo de estructuras en la costa sur de la laguna Mar Chiquita, el sureste de Santiago del Estero y en la región Chaqueña, lo que nos sugiere una posible práctica compartida por estas poblaciones. Estos depósitos han sido relevados en la costa de la Laguna Mar Chiquita y presentan termoalteraciones en sus paredes, lo que podría indicar, más allá del almacenamiento de alimentos, la cocción u otro proceso que implique altas temperaturas (Fabra et al., 2009)4. Métraux (1996) mencionó la utilización de pozos subterráneos en donde se secaban pescados y otros alimentos para su conservación en la región chaqueña, similares a los encontrados en las llanuras del Noreste de la provincia de Córdoba, la presencia de las llamadas "botijas" podrían haber tenido ese mismo propósito. Un estudio etnográfico llevado adelante por Figueroa y Dantas (2006) en donde se describen las técnicas de recolección, procesado y almacenamiento de frutos silvestres en el Noroeste argentino, destaca la presencia de pozos cavados en la tierra en donde se guarda la cosecha junto con cenizas para prevenir la presencia de insectos y humedad. Consideramos que quizás la ingesta de alimentos fibrosos, como las chauchas de algarroba, así como las técnicas de preparación de los alimentos, secado o salado de alimentos, y la posible incorporación de partículas abrasivas externas hayan contribuido al incremento de las tasas de desgaste oclusal en la región Llanuras, más que la introducción de alimentos cultivados y/o más blandos. Finalmente, la presencia de individuos jóvenes y medios con avanzado desgaste a edades tempranas nos estaría indicando que los procesos de estrés masticatorio se iniciaban mucho antes en esta región.

Por otro lado, la presencia estadísticamente significativa de grados de desgaste moderados en Sierras hacia momentos posteriores a los 1200 años AP podría relacionarse con el consumo de alimentos que estuvieran sometidos a técnicas de preparación y cocción que faciliten su ingesta. López (2015) sugiere, en base a las características diferenciales de los distintos tipos cerámicos hallados en el área serrana, el empleo de una gran variedad de formas de cocción, entre ellas el hervido para el preparado de guisos, además del asado, como prácticas habituales hacia momentos finales del Holoceno tardío, que habrían facilitado la digestibilidad de los alimentos y por lo tanto una menor presión masticatoria. En esta región la presencia de grados de desgaste grave se registra a edades avanzadas y los individuos jóvenes presentan menores grados de desgaste que en las Llanuras. Sin embargo debemos remarcar el escaso número de muestras de las categorías etarias adultos jóvenes y mayores para toda la muestra, y la dificultad de poder estimar con certeza la edad de muchas de las muestras, lo que dificulta poder realizar estimaciones poblaciones con mayor confiabilidad.

En cuanto al patrón o forma del desgaste dental, registramos que las formas planas son las predominantes en toda la muestra, así como las cóncavas en primeros y 
segundo molares. Si bien es usual considerar al desgaste como un indicador del tipo de dieta, también destacamos que la variedad en las formas y su dirección pueden estar indicando la realización de actividades paramasticatorias (Belcastro et al., 2007; Larsen, 1985; Molnar, 2008; Waters-Rist, Bazaliiskii, Weber, Goriunova y Katzenberg, 2010). En función de los resultados obtenidos en este trabajo, consideramos que la mayor incidencia de desgastes oblicuos hacia bucal en mandíbula, y oblicuos hacia lingual en maxilar superior, puede responder a la oclusión normal o esperada. Sin embargo, la presencia de desgastes redondeados en la dentición anterior de individuos masculinos y la mayor incidencia de formas oblicuas en la dentición anterior de femeninos, ambos en la región Llanuras, pueden estar indicando la realización de actividades no relacionadas directamente con la ingesta o el procesamiento de alimentos o incluso el masticado de alguna hierba y/o planta (Turner II y Machado, 1983). En la Figura 4A se observan los patrones de desgaste en grupos aborígenes costeros del sur de Brasil (Turner II y Machado, 1983). Estas autoras relacionan la atrición de la superficie lingual de los dientes anteriores del maxilar (LSAMAT siglas en inglés) con el procesamiento y pelado de alimentos vegetales altamente abrasivos y cariogénicos como la mandioca (Manihot esculenta). El patrón de desgaste oblicuo hacia lingual predominante en el maxilar inferior de los individuos relevados en este trabajo está mayormente relacionado con la utilización de la dentición en actividades paramasticatorias antes que con la dureza de los alimentos. En este sentido queremos destacar la gran variabilidad en los patrones de desgaste dental presentes a nivel individual, registrándose formas cóncavas y planas en dentición posterior, y mayor variabilidad en los dientes anteriores con formas oblicuas y redondeadas que en los dientes anteriores, donde también se registra la presencia de surcos oclusales.

Si bien no poseemos información de fuentes etnográficas o históricas sobre la utilización de la dentición en la fabricación de utensilios para las Llanuras, sí se dispone de información etnohistórica sobre los recursos alimenticios y de algunas prácticas cotidianas llevadas adelante por parte de aborígenes de la región chaqueña, particularmente en grupos abipones y mocovíes (Dobrizhoffer, 1968; Métraux, 1996; Paucke, 1942, 1943, 1944). La región Noreste de Córdoba, particularmente el norte de la Laguna Mar Chiquita y los bañados del río Dulce, fueron territorio de incursiones de pueblos abipones durante el siglo XVII, llegando a instalarse una reducción con el nombre de Purísima Concepción de la Nueva Reducción de los Abipones (Dobrizhoffer, 1967, 1968, 1970). Si bien en las fuentes de Paucke $(1942,1943,1944)$, Dobrizhoffer, $(1967,1968,1970)$ y Métraux (1996) no se explicita la utilización de la cavidad bucal como herramienta o "tercera mano" ni el mascado de hierbas u otras sustancias, sí nos relatan distintas técnicas de cocción y elaborado de alimentos, como la preparación de semillas y carne al rescoldo - cocinada sobre cenizas calientes- y de la elaboración de charque o carne seca y de pescados secos. Informan asimismo acerca del consumo de panes hechos con harina de algarroba y de la producción de bebidas fermentadas, así como sobre ciertas restricciones en el consumo de pescado y de bebidas alcohólicas por parte de las mujeres mocovíes y abiponas (Rosso, 2015), junto con prohibiciones temporales particularmente relacionados con ritos de pasajes - pubertad y maternidad(Rosso, 2015). En estas fuentes, la referencia al consumo de algarroba y chañar como recursos centrales en la dieta y las referencias a "borracheras" o festividades realizadas en ocasiones especiales nos remite a la información obtenida de las fuentes históricas para la región serrana ${ }^{5}$, donde se hace mención de estas prácticas con similitudes a las realizadas por pueblos chaqueños durante los siglos XVII y XVIII. Consideramos que la evidencia proporcionada por los estudios arqueológicos (Aparicio, 1933; Bonofiglio, 2010), bioarqueológicos (Fabra et al., 2012a; Fabra et al., 2014a, Fabra, Salega, González, Tavarone, 2014b), arqueomalacológicos (Fabra y Gordillo, 2014; Fabra, Gordillo y Piovano, 2012b; Gordillo y Fabra, 2014), genéticos (Nores, Fabra, García y Demarchi, 2017) y etnohistóricos (Castro Olañeta, 2006) apuntan a la existencia de contactos frecuentes y relaciones biológicas entre los pobladores del noreste de Córdoba con
5. Crónica de Sotelo de Narváez de 1583; Relación Anónima de 1573. 
6. Se prevén estudios sobre microdesgaste dental que podrían contribuir a profundizar las causas de dichas fracturas. poblaciones chaqueñas varios siglos previos al contacto hispano-indígena. Podríamos pensar en prácticas compartidas y formas de vida similares entre estas poblaciones, que implicaron el consumo de algunos alimentos, como así también la realización de actividades diarias como la confección de herramientas o utensilios en donde la cavidad oral era utilizada como herramienta, lo cual podría explicar la variabilidad en las formas del desgaste registradas en esta región.

En síntesis, los grados y patrones de desgaste relevados en este trabajo y las diferencias observadas en momentos tardíos, sobre todo el aumento de los desgastes avanzados en las Llanuras, sugerirían que la variabilidad en las formas y patrones registrados en el desgaste dental de estas poblaciones son producto tanto del uso del aparato masticatorio vinculado a la ingesta de alimentos, como así también actividades paramasticatorias (Belcastro et al., 2007; Molnar, 2008; Turner II y Machado, 1983).

Podemos sugerir que algunos de los individuos femeninos realizaban algún tipo de actividad que implicaba la utilización de la dentición anterior y, particularmente, la superficie lingual de incisivos y caninos, en mayor medida que sus pares masculinos. Los individuos masculinos, por su parte, presentaron un predominio de desgastes redondeados en la dentición posterior, posiblemente debido al desgaste generado por el tipo de oclusión en molares aunque propiciado por el tipo de alimentación y posiblemente con la realización de actividades.

Finalmente en las Sierras, las formas de desgaste predominante son las planas y las cóncavas, sobre todo en molares y premolares. Con relación a ello debemos mencionar que en esta región se registraron las prevalencias más altas de fracturas premortem, particularmente en molares (González, 2016), las cuales pueden estar relacionadas con la introducción de sustancias exógenas, como arena o pequeñas piedras, posiblemente producto de la molienda con herramientas de piedra más que con la realización de actividades extramasticatorias ${ }^{6}$ (Bernal et al., 2007; Gheggi, 2012).

\section{Conclusiones}

A partir del análisis de estos indicadores hemos podido comprobar que la totalidad de los individuos que componen la muestra presentan elevados grados de desgaste dental, con predominancia de formas planas y cóncavas, lo cual sugeriría que estas poblaciones sufrieron altas presiones masticatorias a lo largo de todo el Holoceno tardío. Sin embargo, se registraron algunas diferencias regionales, así se observó que los individuos de la región de Llanuras son los que exhiben los grados de desgaste más avanzados, llegando a afectar la funcionalidad de la pieza dental, sobre todo en individuos masculinos y presentándose a edades más tempranas que en las Sierras. Consideramos que la ingesta de alimentos fibrosos junto a las técnicas de preparación, secado o salado de alimentos, sumado a esto la posible incorporación de partículas abrasivas externas contribuyeron al incremento de las tasas de desgaste oclusal en Llanuras.

Por otro lado en las Sierras se registra una disminución en los grados de desgaste hacia momentos finales del Holoceno tardío. Esto podría estar relacionado con la incorporación de alimentos cultivados como el maíz, según indican los valores de isótopos estables obtenidos para esta región, y el desarrollo de nuevas técnicas de preparación y cocción de los alimentos, que facilitarían su digestibilidad y por ende una disminución en el estrés masticatorio. Consideramos que los grados de desgaste más avanzados registrados en la presente muestra para todo el Holoceno tardío se condicen con lo esperado para poblaciones con un modo de vida basado en una economía de tipo mixta. La disminución del grado de desgaste registrado hacia momentos finales 
estaría indicando una modificación en los modos de preparación y cocción de los alimentos, vinculada a nuevas formas de adquirir y procesar alimentos, con menor estrés masticatorio sobre todo en la región de Sierras, tal como sugieren los datos isotópicos y bioarqueológicos.

En cuanto al patrón de desgaste dental, las formas oblicuas hacia bucal en mandíbula y oblicuas hacia lingual en maxilar superior pueden responder a la oclusión normal o esperada. Sin embargo, se detectaron facetas de desgaste anómalas: la presencia de formas redondeadas en la dentición anterior de individuos masculinos y la mayor incidencia de formas oblicuas en la dentición anterior de femeninos, ambos en la región Llanuras, pueden estar indicando la realización de actividades no relacionadas directamente con la ingesta o el procesamiento de alimentos. En Sierras, la menor presencia de facetas anómalas y la mayor incidencia de patrones de desgaste cóncavos y planos tiene una asociación más directa con el procesamiento de los alimentos, particularmente la utilización de morteros o molinos de piedra los cuales pueden desprender partículas que aceleren la pérdida de esmalte dental y provoquen un "ahuecamiento" o cupping de la dentina, sobre todo en molares y premolares y tal como lo indican la presencia de fracturas premortem en molares en esta región (González, 2016).

El estudio de las formas y los grados de desgaste dental, realizado por primera vez desde una perspectiva poblacional, permitió sumar otra línea de evidencia a la ofrecida por las patologías orales y los estudios isotópicos ya desarrollados en esta misma muestra, para aportar información no solo sobre dieta y alimentación sino también sobre otras prácticas sociales. Se pretende continuar y profundizar estos estudios a través del análisis del microdesgaste dental, para estimar el tipo de dieta consumida, las texturas de los alimentos -básicamente la presencia de fibras- y el grado de dureza de los componentes de la dieta, $y$ las fuentes potenciales de partículas abrasivas introducidas por las técnicas de preparación de los alimentos o las prácticas culturales y así estimar con mayor certeza el origen de las formas de degaste observadas.

\section{Agradecimientos}

Las autoras desean agradecer a los museos públicos que facilitaron el acceso a sus colecciones: Museo de Antropología (FFyH, UNC), Museo Histórico Municipal de La Para (La Para, Córdoba), Museo de la región de Ansenuza "Aníbal Montes" (Miramar, Córdoba) y Museo Arqueológico Provincial "Aníbal Montes” (Río Segundo, Córdoba). También al Dr. Darío Demarchi y al Dr. Mario Arrieta por su asesoramiento en la realización de los análisis estadísticos. Finalmente a los evaluadores anónimos del trabajo, quienes con sus sugerencias y comentarios permitieron mejorar la versión originalmente presentada. Este trabajo contó con el financiamiento de FONCyT (PICT 2013-2028) y SECyT-UNC (2014-2015). 


\section{Q Referencias citadas}

» Alt, K. W. y Pilcher, S. (1998). Artificial modifications of human teeth. En K. W. Alt., F.W. Rosing y M. Teschler-Nicola (Eds.), Dental Anthropology, Fundamentals, Limits and Prospects (pp.387-415). Springer: Berlin.

"Aparicio, F. D. (1933). La antigua provincia de los Comechingones (Volumen 1). Buenos Aires: Junta de Historia y Humanística de América.

» Belcastro, G., Rastelli, E., Mariotti, V., Consiglio, C., Facchini, F. y Bonfiglioli, B. (2007). Continuity or Discontinuity of the Life-Style in Central Italy During the Roman Imperial Age-Early Middle Ages Transition: Diet, Health, and Behavior. American Journal of Physical Anthropology, 132, 381-394.

» Bernal, V., Novellino, P., González, P. y Pérez, S. I. (2007). Role of Wild Plant Foods among Late Holocene Hunter-Gatherers From Central and North Patagonia (South America): An Approach From Dental Evidence. American Journal of Physical Anthropology, 133, 1047-1059.

"Bonofiglio, M. (2010). Las comunidades productoras de alimentos de la llanura. En E. Berberían (Ed.) Los pueblos indígenas de Córdoba (pp. 93-101). Córdoba: Centro de estudios Históricos “Dr, Carlos Segretti”.

" Bonfiglioli, B., Mariotti, V., Facchini, F., Belcastro, M. G. y Condemi, S. (2004). Masticatory and non-masticatory dental modifications in the Epipalaeolithic Necropolis of Taforalt (Morocco). International Journal of Osteoarchaeology, 14, 448-456.

"Buikstra, J. y Ubelaker, D. (1994). Standards for data collection from human skeletal remains. Faytteville: Arkansas Archaeological Survey Research Series № 44.

»Cabrera, A. L. (1976). Regiones fitogeográficas argentinas. Enciclopedia Argentina de Agricultura y Jardinería (Tomo II, Fascículo 1) (pp.1-85). Buenos Aires: Editorial Acme.

"Campillo. D, (2001). Introducción a la Paleopatología. Barcelona: Ediciones Balleterra.

"Castro Olañeta, I. (2006). Transformaciones y continuidades de las sociedades indígenas en el sistema colonial. El pueblo de indios de Quilino a principios del siglo XVII. Córdoba: Alción Editora.

" Clarke, N. G. y Hirsch, R. S. (1991). Tooth Dislocation: the relationship with tooth wear and dental abscesses. American Journal of Physical Anthropology, 85, 293-298.

»Clement, A. F. y Hillson, S. W. (2013). Macro tooth wear patterns amongst the early hominins of South Africa. En Proceedings of the 82nd Annual Meeting of the American Association of Physical Anthropologists (pp. 99). Hoboken: Wiley-Blackwell.

"Cornero, S., Del Río, P. y Ceruti, C. (2013). Sitios con "hornitos" del Holoceno Tardío en el chaco austral. Colonia Dolores, depto. San Justo, Pcia. de Santa Fe. Anuario de Arqueología (UNR), 5, 103-115.

"Cruwys, E. (1989). Tooth Wear and the Archaeologist. The role of human tooth wear in archaeological research. En C. A. Roberts, F. Lee y J. Bintliff (Eds.), Burial Archaeology Current Research Methods and Development (pp. 251-166). Oxford: BAR, British Series 211.

" Cruwys, E., Robb, N. D. y Smith, B. (1992). Anterior tooth notches: an Anglo-Saxon case of study. Journal of Paleopathology, 4(3), 211-220.

»Delgado-Darías, T., Velasco-Vázquez, J., Arnay de Larosa, M., Martín-Rodríguez, E. y González-Reimers, E. (2005). Dental caries among the prehispanic population from Gran Canaria. American Journal of Physical Anthropology, 128, 560-568. 
» Deter, C. A. (2009). Gradients of occlusal wear in hunter-gatherers and agriculturalists. American Journal of Physical Anthropology, 138, 247-254.

»Dobrizhoffer, M. (1967). Historia de los Abipones (Volumen I). Resistencia: Universidad Nacional de Noroeste.

»Dobrizhoffer, M. (1968). Historia de los Abipones (Volumen 2). Resistencia: Universidad Nacional de Noroeste.

»Dobrizhoffer, M. (1970). Historia de los Abipones (Volumen 3). Resistencia: Universidad Nacional de Noroeste.

» Fabra, M. y Demarchi, D. (2013). Análisis morfogeométrico aplicado al estudio de los patrones espaciales y temporales de variación morfológica craneofacial en poblaciones del centro de Argentina. Cuadernos del Instituto Nacional de Pensamiento Latinoamericano. Series Especiales, 1(1), 87-101

» Fabra, M. y González, C. V. (2015). Diet and oral health of populations that inhabited central Argentina (Córdoba province) during late Holocene. International Journal of Osteoarchaeology, 25, 160-175.

»Fabra, M. y Gonzalez, C. V. (2016). Salud oral, dieta y cambio social en poblaciones de la Región Central de Argentina a lo largo del Holoceno. Trabajo presentado en el XIX Congreso Nacional de Arqueología Argentina, Tucumán, Argentina.

» Fabra, M., González, C. y Salega, S. (2012a). Modos de vida e historia biológica de poblaciones de las Sierra y Llanuras de Córdoba (Argentina): aproximaciones desde el registro bioarqueológico. Revista Argentina de Antropología Biológica, 14, 87-104.

»Fabra, M. y Gordillo, S. (2014). Estimaciones acerca del uso de una almeja de agua dulce (Diplodon parallelopipedon) hallada en contexto arqueológico en el Mar de Ansenuza (Córdoba, Argentina). En H. Hammond y M. A. Zubimendi (Eds.), Arqueología y malacología: abordajes metodológicos y casos de estudio en el Cono Sur (pp. 129-143). Ciudad Autónoma de Buenos Aires: Fundación de Historia Natural Félix de Azara.

»Fabra, M., Gordillo, S. y Piovano, E. L. (2012b). Arqueomalacología en las costas de Ansenuza: análisis de una almeja nacarífera (Anodontites trapesialis) hallada en contexto funerario del sitio El Diquecito (Laguna Mar Chiquita, Córdoba). Arqueología, 18, 257266.

» Fabra, M., Nores, R., Salega, S. y González, C. V. (2014a). Entre las sierras y el Mar: investigaciones bioarqueológicas en el noroeste de la región pampeana (costa sur Laguna Mar Chiquita, Córdoba, Argentina). En L. Luna, C. Aranda y J. Suby (Eds,), Avances recientes en la Bioarqueología Sudamericana (pp. 205-230). Buenos Aires: Grupo de Investigación en Bioarqueología.

»Fabra, M., Salega, S. y González, C. V. (2009). Comportamiento mortuorio en poblaciones prehispánicas de la región austral de las Sierras Pampeanas durante el Holoceno. Arqueología, 15, 165-186.

»Fabra, M., Salega, S., González, C. V. y Tavarone, A. (2014b). Lo que el agua nos dejó: investigaciones bioarqueológicas en la costa sur de la laguna Mar Chiquita (Córdoba, Argentina). Jangwa Pana, Revista del Programa de Antropología, 13, 51-64.

»Figueroa, G. y Dantas, M. (2006). Recolección, procesamiento y consumo de frutos silvestres en el noroeste semiárido argentino, Casos actuales con implicancias arqueológicas. La Zaranda de Ideas, 2, 35-50.

»Flensborg, G. (2011). Dento-alveolar lesions and palaeodietary inferences from the Paso Alsina 1 site (eastern Pampean-Patagonian transition, Argentina). Journal of Comparative Human Biology, 65(5), 335-350. 
» Flensborg, G. (2012). Análisis paleopatológico en el curso inferior del río Colorado (Pcia, De Buenos Aires). Exploración y evaluación del estado de salud de sociedades cazadoras recolectoras en el Holoceno tardío. (Tesis Doctoral inédita), Universidad Nacional del Centro de la Provincia de Buenos Aires, Argentina.

»Frenguelli, J. y De Aparicio, F. (1932). Excursión a la Laguna de Mar Chiquita (Provincia de Córdoba). Publicaciones del Museo Antropológico y Etnográfico de la Facultad de Filosofía y Letras, Serie A(II), 121-166.

" Gheggi, M. S. (2012). Patologías orales, dieta y modo de vida en Esquina de Huajra (Quebrada de Humahuaca, Jujuy). Revista Argentina de Antropología Biológica, 14(1), 6577 .

» Gilbert, B. y Mckern, T. (1973). A method for aging the female Os pubis. American Journal of Physical Anthropology, 38, 31-38.

» González C. V. (2011). Dieta y salud oral de las poblaciones prehispánicas de la región austral de las sierras pampeanas: aportes desde la antropología dental. (Tesis de Licenciatura inédita), Universidad Nacional de Córdoba, Argentina.

» González, C. V. (2016). Dieta y salud oral en poblaciones del centro de Argentina durante el Holoceno tardío. (Tesis Doctoral inédita), Universidad Nacional de Córdoba, Argentina.

»González, C. V. y Fabra, M. (2011). Estimaciones acerca de la salud de poblaciones que ocuparon la región austral de las sierras pampeanas en el holoceno tardío: una aproximación desde la Antropología Dental. Revista del Museo de Antropología, 4, 161-178.

» González Galán, A., Correa, A., Patto De Abreu, C. M. y Piccolo Barcelos, M. F. (2008). Caracterización química de la harina del fruto de Prosopis spp, procedente de Bolivia y Brasil. ALAN. Archivos Latinoamericanos de Nutrición, 58(3), 309-315.

"Gómez Otero, J. y Novellino, P. (2011). Diet, nutritional status and oral health in huntergatherers from the Central-Northern coast of Patagonia and the Chubut river lower valley, Argentina. International Journal of Osteoarchaeology, 21, 643-659.

»Gordillo, S. y Fabra, M. (2014). El uso de moluscos y caracoles por parte de poblaciones prehispánicas que habitaron el Mar de Ansenuza en el norte cordobés. Memorias del Pueblo Digital: Revista del Museo Histórico Municipal de La Para, 1, 5-17.

» Hillson, S. (1996). Dental Anthropology. Cambridge: Cambridge University Press.

» Hinton, R. J. (1981). Form and patterning of anterior tooth wear among aboriginal human groups. American Journal of Physical Anthropology, 54, 555-564.

" Krenzer, U. (2006). Compendio de métodos antropológico forenses para la reconstrucción del perfil osteo biológico. Guatemala: Centro de Análisis Forense y Ciencias Aplicadas.

"Laguens, A. (1999). Arqueología del contacto hispano-indígena. Un estudio de cambios y continuidades en las Sierras Centrales de Argentina. Oxford: BAR International Series 801, Archaeopress.

"Laguens, A. y Bonnin, M. (1987). Espacio, paisaje y recursos, Estrategias indígenas alternativas y complementarias en la cuenca del río Copacabana (Dto, Ischilín, Córdoba, Arg,), Sitio El Ranchito: 1000 aC-16oo dC. Publicaciones del Instituto de Antropología, XLV, 159-201.

»Laguens, A. y Bonnin, M. (2009). Sociedades indígenas de las Sierras Centrales. Arqueología de Córdoba y San Luis. Córdoba: Universidad Nacional de Córdoba.

»Laguens, A., Fabra, M., Macedos Dos Santos, G. y Demarchi, D. (2009). Paleodietary inferences based on isotopic data for pre-hispanic populations of the Central Mountains of Argentina. International Journal of Osteoarchaeology, 19, 237-249. 
"Larsen, C. S. (1985). Dental Modifications and tool use in the Western Great Basin. American Journal Physical Anthropology, 67, 393-402.

» Larsen, C. S., Teaford, M. F. y Sandford, M. K. (1998). Teeth as tools at tutu: extramasticatory behavior in prehistoric St. Thomas, U,S. Virgin Islands. En J. R. Lucaks (Ed.), Human dental development, morphology and pathology: A tribute to Albert Dahlberg (pp. 402-420). Eugene: University of Oregon.

» Lev-Tov, C. y Smith, P. (2006). Variation in occlusal dental wear of two chalcolithic populations in the Southern Levant. American Journal of Physical Anthropology, 130(4), 471- 479 .

»L'Heureux, G. (2000). Estudio comparativo de indicadores de adecuación fisiológica y salud bucal en muestras de restos humanos del sudeste de la región pampeana. Relaciones de la Sociedad Argentina de Antropología, XXV, 51-73.

»L'Heureux, G. (2002). Inferencias paleodietarias a partir del análisis de los patrones de desgaste dental y frecuencia de caries en muestras de restos humanos del Holoceno del sudeste de la Región Pampeana. En D. Mazzanti, M. Berón y F. Oliva (Eds.), Del Mar a Los Salitrales, 10000 años de historia pampeana en el umbral del tercer milenio (pp. 127-140). Mar del Plata: Universidad Nacional de Mar del Plata.

» Littleton, J. y Frohlich, B. (1993). Fish-eaters and farmes: dental pathology in the Arabian Gulf. American Journal of Physical Anthropology, 92, 427-447.

» López, M. L. (2015). Prácticas culinarias como medio para la reproducción social de los grupos prehispánicos de las sierras de Córdoba. En Julián Salazar (Comp.), Condiciones de posibilidad de la reproducción social en sociedades prehispánicas y coloniales tempranas en las Sierras Pampeanas (República Argentina) (pp. 117-212). Córdoba: Centro de Estudios Históricos Prof. Carlos S. A. Segreti.

» López, M. L., Medina, M. E. y Rivero, D. E. (2015). First records of Chenopodium spp./ Amaranthus spp. starch grains and their relevance to the study of the Late Holocene human subsistence in Central Argentina. The Holocene, 25(2), 288-296.

» Lovejoy, C., Meindl, R., Mensforth, R. y Barton, T. (1985). Multifactorial determination of skeletal age at death: A method and blind tests of its accuracy. American Journal of Physical Anthropology, 68, 1-14.

»Lozano, M. (2005). Estudio del desgaste a nivel microscópico de los dientes anteriores de los homínidos del yacimiento pleistocénico de Sima de los Huesos (Sierra de Atapuerca, Burgos. (Tesis Doctoral inédita), Universitat Rovira i Virgili, España.

» Lukacs, J. R. y Pastor, R. F. (1988). Activity-induced patterns of dental abrasion in prehistoric Pakistan: Evidence from Mehrgarh and Harappa. American Journal of Physical Anthropology, 76, 377-398.

» Luna, L. y Aranda, C. (2014). Trends in oral pathology of hunter-gatherers from Western Pampas, Argentina. Anthropological Science, 122(2), 55-67.

» Medina, M. (2009).Tendencias en el consumo prehispánico tardío de recursos faunísticos: Zooarqueología de C.Pun.39 y Puesto La Esquina 1 (Córdoba, Argentina). Archaeofauna, 18, 119-136.

» Medina, M., López, L. y Berberian, E. (2009). Agricultura y recolección en el tardío prehispánico de las Sierras de Córdoba (Argentina). El registro arqueobotánico de C. Pun.39. Arqueología, 15, 217-229.

» Meindl, R. y Lovejoy, C. (1985). Ectocranial suture closure: a revised method for the determination of skeletal age at death based on the lateral anterior sutures. American Journal of Physical Anthropology, 68, 57-66. 
» Menéndez, L. (2010). Patologías bucales en cráneos humanos del noreste de Patagonia: tendencias temporales en la dieta y estado de salud de las poblaciones del Holoceno tardío del Valle inferior de Rio Negro. Magallania, 38(2), 115-126.

» Métraux, A. (1996). Etnografía del Chaco. Paraguay: Editorial El Lector.

» Molnar, S. (1971). Human tooth wear, tooth function and cultural variability. American Journal of Physical Anthropology, 34, 175-190.

» Molnar, S. (1972). Tooth wear and culture: a survey of tooth functions among some prehistoric populations. Current Anthropology, 13, 511-526.

» Molnar, P. (2008). Dental wear and oral pathology: possible evidence and consequences of habitual use of teeth in a Swedish Neolithic sample. American Journal of Physical Anthropology, 136, 423-431.

" Neto Wasterlain, R. S. (2006). "Males da boca”, Estudo da patología numa amostra das Coleccoes ostelógicas identificadas do Museu Antropologico da Universidade da Coimbra (finais du sec, XIX/sec, XX). (Tesis Doctoral inédita), Universidad de Coimbra, Portugal.

» Nores, R. y Demarchi, D. A. (2011). Análisis de haplogrupos mitocondriales en restos humanos de sitios arqueológicos de la provincia de Córdoba. Revista Argentina de Antropología Biológica, 13(1), 43-54.

» Nores, R., Fabra, M. y Demarchi, D. A. (2011). Variación temporal y espacial en poblaciones prehispánicas de Córdoba, Análisis de ADN antiguo. Revista del Museo de Antropología, 4, 187-194.

» Nores, R., Fabra, M., García, A. y Demarchi, D. (2017). Diversidad genética en restos humanos arqueológicos del sitio El Diquecito (Costa sur, Laguna Mar Chiquita, Provincia de Córdoba). Revista Argentina de Antropología Biológica, 19(1), 1-12.

" Pastor, S. (2007). Arqueología del Valle de Salsacate y pampas de altura adyacentes (Sierras Centrales de Argentina). Una aproximación a los procesos sociales del período prehispánico tardío (900-1573 d.C.). (Tesis Doctoral inédita), Universidad Nacional de La Plata, Argentina.

» Pastor, S. y Lopez, L. (2010). Consideraciones sobre la agricultura prehispánica en el sector central de las Sierras de Córdoba (Argentina). En A. Korstanje y M. Quesada (Eds.), Arqueología de la Agricultura: Casos de Estudios en la Región Andina Argentina (pp. 208-233). Catamarca: Editorial de la Universidad Nacional de Catamarca.

» Paucke, F. (1942). Hacia Allá y para Acá. Una Estadía entre los Indios Mocobíes, 17491767. (Volumen I). Tucumán: Universidad Nacional de Tucumán.

» Paucke, F. (1943). Hacia allá y para acá. Una estadía entre los indios Mocobíes, 1749-1767. (Volumen II). Tucumán: Universidad Nacional de Tucumán.

»Paucke, F. (1944). Hacia allá y para acá. Una estadía entre los indios Mocobíes, 1749-1767. (Volumen III). Tucumán: Universidad Nacional de Tucumán.

» Piana de Cuestas, J. (1992). Los indígenas de Córdoba bajo el régimen colonial (1570-1620). Córdoba: Dirección General de Publicaciones de la Universidad Nacional de Córdoba.

» Powell, M. (1985). The analysis of dental wear caries for dietary reconstruction. En R. I. Gilbert y J. H. Mielke (Eds.), The Analysis of Prehistoric Diet (pp. 307-358). Orlando: Academic Press.

» Relación Anónima (1573). En C. Segretti. (Comp.), Relación de la tierra nueva que don Jerónimo de Cabrera, gobernador de Tucumán, descubrió en aquella provincia, Córdoba, ciudad y provincia (pp. 13-15). Córdoba: Junta Provincial de Historia. 
» Rivero, D., Medina, M., Recalde, A. y Pastor, S. (2010). Variabilidad en la explotación de recursos faunísticos durante el Holoceno en las Sierras de Córdoba (Argentina): una aproximación zooarqueológica. En M. Gutiérrez, M. De Nigris, P. Fernández, M. Giardina, A. Gil, A. Izeta, G. Neme y H. Yacobaccio (Eds.), Zooarqueología a principios del siglo XXI: aportes teóricos, metodológicos y casos de estudio (pp. 321-332). Buenos Aires: Libros del Espinillo.

» Rosso, C. (2015). Las celebraciones indígenas desde una perspectiva etnobotánica histórica: el caso de los mocovíes de la reducción de San Javier durante el siglo XVIII. Chungara. Revista de Antropología Chilena, 4, 645-658.

»Salega, S. y Fabra, M. (2013). Niveles de actividad física en poblaciones de las sierras y las Ilanuras de la provincial de Córdoba (Argentina) durante el Holoceno tardío. Relaciones de la Sociedad Argentina de Antropología, XXXVIII(2), 401-420.

» Scott, E. C. (1979). Dental wear scoring technique. American Journal of Physical Anthropology, 51, 213-218.

» Smith, B. H. (1984). Patterns of molar wear in hunter-gartherers and agriculturalists. American Journal of Physical Anthropology, 63, 39-56.

» Soames, J. V. y Southam, J. C. (2005). Oral pathology. Oxford: Oxford University Press.

» Sotelo De Narváez, P. (1583). Relación de las Provincias de Tucumán que dió P Sotelo de Narváez, vecino de aquellas provincias, al muy ilustre Sr, Lic, Cepeda. Relaciones Geográficas de Indias. España: Ministerio de Fomento.

»Todd, T. (1921). Age changes in the pubic bone, I: the male white pubis. American Journal of Physical Anthropology, 3, 285-334.

» Turner II, C. G. y Machado, L. M. C. (1983). A new dental wear pattern and evidence for high carbohydrate consumption in a Brazilian archaic skeletal population. American Journal of Physical Anthropology, 61, 125-130.

»Waters-Rist, A., Bazaliiskii, V., Weber, A., Goriunova, O. y Katzenberg, M. A. (2010). Activity-induced dental modification in Holocene siberian hunter-fisher-gatherers. American Journal of Physical Anthropology, 143, 266-278. 